\title{
Nitrogen, Phosphorus, and Potassium Adsorption and Desorption Improvement and Soil Buffering Capacity Using Clinoptilolite Zeolite
}

\author{
Perumal Palanivell ${ }^{1}$, Osumanu Haruna Ahmed ${ }^{2,3,4, *}$, Latifah Omar ${ }^{2,3}$ and Nik Muhamad Abdul Majid 5 \\ 1 PPB Oil Palms Berhad, Lot 1A, KM 15, Labuk Road, Sandakan 90000, Sabah, Malaysia; devend05@gmail.com \\ 2 Department of Crop Science, Faculty of Agriculture and Forestry Sciences, Bintulu Sarawak Campus, \\ Universiti Putra Malaysia, Bintulu 97008, Sarawak, Malaysia; latifahomar@upm.edu.my \\ 3 Institut Ekosains Borneo (IEB), Universiti Putra Malaysia, Bintulu 97008, Sarawak, Malaysia \\ 4 Institute of Tropical Agriculture and Food Security (ITAFoS), Universiti Putra Malaysia, \\ Serdang 43400, Selangor, Malaysia \\ 5 Institute of Tropical Forestry and Forest Product (INTROP), Universiti Putra Malaysia, \\ Serdang 43400, Selangor, Malaysia; nik@upm.edu.my \\ * Correspondence: osumanu@upm.edu.my; Tel.: +60-86-855406; Fax: +60-86-855415
}

check for updates

Citation: Palanivell, P.; Ahmed, O.H.; Omar, L.; Abdul Majid, N.M. Nitrogen, Phosphorus, and Potassium Adsorption and Desorption Improvement and Soil Buffering Capacity Using Clinoptilolite Zeolite. Agronomy 2021, 11, 379. https://doi.org/10.3390/ agronomy11020379

Academic Editor: Diego Pizzeghello

Received: 15 January 2021

Accepted: 17 February 2021

Published: 20 February 2021

Publisher's Note: MDPI stays neutral with regard to jurisdictional claims in published maps and institutional affiliations.

Copyright: (c) 2021 by the authors. Licensee MDPI, Basel, Switzerland. This article is an open access article distributed under the terms and conditions of the Creative Commons Attribution (CC BY) license (https:/ / creativecommons.org/licenses/by/ $4.0 /)$.

\begin{abstract}
The physical and chemical properties of clinoptilolite zeolite can be used to enhance soil nutrient availability for optimum crop use. Amending nitrogen, phosphorus, and potassium fertilizers with clinoptilolite zeolite could create a pool of negative charges to retain and release nutrients timely for crop use. Thus, we used clinoptilolite zeolite to enhance Typic Paleudults sorption (adsorption and desorption) of nitrogen, phosphorus, potassium, and this soil's pH buffering capacity. The treatments evaluated were: (i) $250 \mathrm{~g}$ soil alone, (ii) $20 \mathrm{~g}$ clinoptilolite zeolite alone, (iii) $250 \mathrm{~g}$ soil +20 g clinoptilolite zeolite, (iv) $250 \mathrm{~g}$ soil $+40 \mathrm{~g}$ clinoptilolite zeolite, and (v) $250 \mathrm{~g}$ soil $+60 \mathrm{~g}$ clinoptilolite zeolite. Clinoptilolite zeolite increased soil nitrogen and potassium adsorption, nitrogen desorption, and soil $\mathrm{pH}$. Moreover, ability of the soil to resist drastic change in $\mathrm{pH}(\mathrm{pH}$ buffering capacity) was improved. Additionally, phosphorus adsorption and desorption of phosphorus and potassium were reduced. Higher potassium adsorption with lower potassium desorption suggests that the clinoptilolite zeolite sorbs potassium effectively. The clinoptilolite zeolite nitrogen, phosphorus, and potassium contributed to the reduction in the adsorption these nutrients. The clinoptilolite zeolite improved nitrogen, phosphorus, and potassium availability and soil buffering capacity to prevent these nutrients from being fixed or lost through for example, leaching. Therefore, clinoptilolite zeolite application could contribute to improved use of nitrogen, phosphorus, and potassium fertilizers to prevent soil, air, and water pollution. Additionally, our intervention could improve nitrogen, phosphorus, and potassium use efficiency.
\end{abstract}

Keywords: ammonium; phosphate; potassium; retention; released; sorption

\section{Introduction}

Nitrogen $(\mathrm{N})$, phosphorus $(\mathrm{P})$, and potassium $(\mathrm{K})$ are important nutrients for crop productivity. These nutrients are made available in soils through sorption processes, such as absorption and adsorption. The mechanism through which positively charges ions adsorbed by the negatively charged parts of soils among others is referred to as adsorption [1,2] whereas the release of adsorbed ions is called desorption [3,4]. Apart from ensuring $\mathrm{NH}_{4}{ }^{+}$retention in soils, adsorption improves $\mathrm{N}$ use efficiency of crops in addition to preventing leaching of $\mathrm{NH}_{4}{ }^{+}$and nitrate $\left(\mathrm{NO}_{3}{ }^{-}\right)$to contaminate water bodies which commonly causes algae bloom. The adsorption of $\mathrm{K}^{+}$using clinoptilolite zeolite (CZ) is similar to $\mathrm{NH}_{4}{ }^{+}$. Thus, the technology for extracting $\mathrm{K}^{+}$from seawater using $\mathrm{CZ}$ as an adsorbent has been adopted into industrial operation [5]. However, for anion such as phosphate $\left(\mathrm{PO}_{4}{ }^{3-}\right)$, surfactant-modified zeolites could be used to remove $\mathrm{P}$ anionic 
through adsorption. Reports from several studies suggest that surfactant-modified zeolite adsorbs anions such as $\mathrm{NO}_{3}{ }^{-}$, sulphate $\left(\mathrm{SO}_{4}{ }^{2}\right)$, chromate $\left(\mathrm{CrO}_{4}{ }^{2-}\right)$, and hydrogen arsenate $\left(\mathrm{HAsO}_{4}{ }^{2-}\right)$ [6], $\mathrm{CrO}_{4}{ }^{2-}[7,8]$, and $\mathrm{HAsO}_{4}{ }^{2-}$ [9].

The nutrients that are adsorbed in soils are readily used by crops than the absorbed ones and Langmuir, Freundlich, and Temkin methods are commonly used to measure these fractions. The amount of nutrients in the form of a single layer on the soil surface is measured using Langmuir isotherm suggesting that this method is suitable for single layer surface adsorption reaction for adsorption sites that are fixed [10,11]. Freundlich isotherm demonstrates how adsorbed nutrients and adsorbent surface are related based on the premise that different sites or heterogeneous adsorbent surface with many adsorption energies exist [12]. The binding energy involved in adsorbing nutrient is measured by Temkin isotherm based on the assumption that heat generation during the process of nutrient adsorption decreases linearly as the adsorbent coverage increases, following the interaction among the adsorbates (uniform binding energy controls how nutrients are adsorbed) $[13,14]$.

Soil buffering capacity is one of the determinant factors that affects adsorptions of nutrients [15]. Initial soil $\mathrm{pH}$, amount of acid or alkali, and soil $\mathrm{pH}$ buffering capacity are the most important factors that control soil $\mathrm{pH}$. Soil $\mathrm{pH}$ resilience is controlled by several chemical reactions such as protonation or deprotonation of acidic compounds and ion exchange [16]. Leaching loss of $\mathrm{N}$ and $\mathrm{K}$ because of poor soil retention has adverse effect on soil acidity. Ultisols and Oxisols are acid soils with $\mathrm{pH} 4$ to 5, less fertile, low in cation exchange capacity (CEC), and high kaolinite and sesquioxides (oxides of Fe and Al). Phosphorus is not available in acid soils partly because of P fixation [17]. However, Ultisols and Oxisols can be productive when limed, fertilized, and conditioned or amended with $\mathrm{CZ}$.

Amending low $\mathrm{pH}$ soils with $\mathrm{CZ}$ improves their $\mathrm{pH}$ and the productivity of crops [18] because of the neutralizing ability of $C Z$, the ion-exchange of $C Z$, and the affinity of $C Z$ for water and positively charge ions [3]. In other related studies, Peres-Caballero et al. [19] described zeolites as crystalline hydrated aluminosilicates whose size ranges from 0.3 to $1 \mathrm{~nm}$ upon being in contact with water and this property has compelled researchers to believe that zeolites qualify as a sieving materials with unique nutrient (for example, $\mathrm{NH}_{4}^{+}$ and $\mathrm{K}^{+}$) selection ability $[19,20]$. The selective nutrient adsorption nature of zeolites also ensures timely release of adsorbed nutrients (for example, $\mathrm{NH}_{4}{ }^{+}$and $\mathrm{K}^{+}$) in a manner is that in synchrony crop needs to guarantee nutrient use efficiency in sustainable farming systems [21-23]. The cation exchange property of zeolites have been exploited to sorb $\mathrm{NH}_{4}^{+}$ and $\mathrm{K}^{+}$from aqueous solution in addition to using the pores of aluminosilicate groups in zeolites to adsorb cations. In terms of soil $\mathrm{P}$ and soil acidity management, capitalizing on the CEC and pH of CZ might not only reverse $\mathrm{P}$ fixation but it will also increase soils $\mathrm{pH}$. Based on these rationales, our premonition is that amending acid soils with $\mathrm{CZ}$ will improve soil $\mathrm{pH}$ resilience, $\mathrm{N}, \mathrm{P}$, and $\mathrm{K}$ availability besides minimizing $\mathrm{P}$ fixation by $\mathrm{Al}$ and Fe.

Although $\mathrm{CZ}$, if used as adsorbent for $\mathrm{NH}_{4}{ }^{+}$and $\mathrm{K}^{+}$, can complement to chemical fertilization and liming programs, the literature is not replete with data on how acid soils could be amended with CZ to significantly improve N, P, and K availability. To this end, this present study addressed the following research questions: (i) Is it possible to use $\mathrm{CZ}$ to amend acid soils' to significantly improve retention of $\mathrm{N}, \mathrm{P}$, and $\mathrm{K}$ in addition to improving soil $\mathrm{pH}$ resilience and, (ii) what is the optimum rate of $\mathrm{CZ}$ that improve acid soils' N, P, and K availability and buffering capacity? To answer the afore-stated research questions, the objectives of this study were to determine the effects of amending Bekenu Series (Typic Paleudults) with CZ on adsorption and desorption of N, P, and K, soil pH, and soil pH buffering capacity. Studies on nutrients adsorption such as N, P, and K and soil $\mathrm{pH}$ buffering capacity using $\mathrm{CZ}$ as soil amendment are essential in determining soil response to $\mathrm{N}, \mathrm{P}$, and $\mathrm{K}$ additions and the capacity to buffer the net inputs of acid. The implications of including $\mathrm{CZ}$ as a soil amendment is an attempt to delay nutrients migration 
out of farms. With our intervention, fertilizer applications following using CZ at optimum amount, leaching loss of nutrients such as N, P, and K could be prevented. This study also provides information on the mechanism of $\mathrm{N}, \mathrm{P}$, and $\mathrm{K}$ adsorb and desorb reflected by the different sorption isotherms and the capacity of Bekenu Series (Typic Paleudults) to buffer the changes in $\mathrm{pH}$ from acidity input.

\section{Materials and Methods}

\subsection{Soil Sampling, Preparation, and Selected Physico-Chemical Analyses}

A Typic Paleudults (Bekenu Series) soil located at Universiti Putra Malaysia Bintulu Sarawak Campus, Malaysia (Figure 1) was sampled at 0 to $25 \mathrm{~cm}$, after which the soil was prepared using standard procedures for the sorption studies. Details about the experimental site has been published in one of our papers [24].

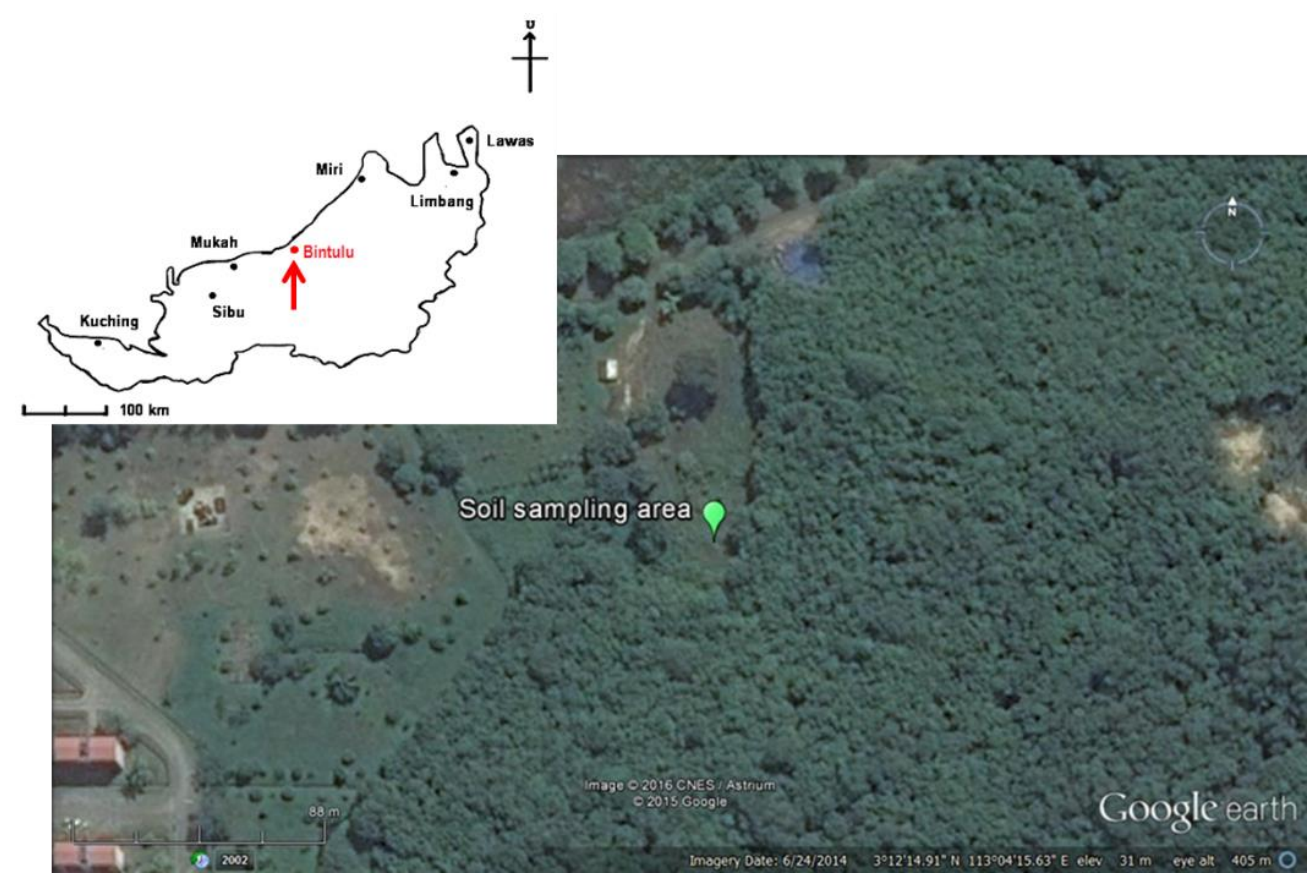

Figure 1. Aerial view showing where the soil used for the sorption studies was taken.

Selected soil physical properties (Table 1) were determined using the method described by Tan [25]. pH of the soil was determined in a ratio of 1:2 (soil: distilled water suspension) using a $\mathrm{pH}$ meter. Soil total $\mathrm{C}, \mathrm{N}$, and organic matter were determined using a Leco carbon, hydrogen, nitrogen, and sulphur (CHNS) Analyzer (LECO Truspec Micro Elemental Analyzer CHNS, New York, NY, USA). Soil available P was extracted using the double acid method [25] followed by the molybdenum blue method [26]. Soil exchangeable $\mathrm{Ca}, \mathrm{Mg}$, and $\mathrm{K}$ were extracted using the leaching method [25] after which the contents of these cations were determined using Atomic Absorption Spectrophotometry (Analyst 800, Perkin Elmer, Norwalk, CT, USA). Soil cation exchange capacity was determined using the leaching method [25] followed by steam distillation. The method of Keeney and Nelson [27] was used to extract exchangeable $\mathrm{NH}_{4}{ }^{+}$and available $\mathrm{NO}_{3}{ }^{-}$after which the concentrations of these ions were determined using steam distillation. The selected physical and chemical properties of the soil (Bekenu Series, Typic Paleudults) that was used in the adsorption and desorption studies are comparable to those reported by Paramananthan [28] except for CEC, sand, silt, and clay contents. The selected chemical properties of the soil are summarized in Table 1. 
Table 1. Physico-chemical characteristics of the soil used in the sorption studies.

\begin{tabular}{|c|c|c|}
\hline Property & Current Study & Range $^{*}(0-36 \mathrm{~cm})$ \\
\hline $\mathrm{pH}$ & 4.41 & $4.6-4.9$ \\
\hline $\mathrm{EC}\left(\mu \mathrm{S} \mathrm{cm} \mathrm{cm}^{-1}\right)$ & 53.90 & NA \\
\hline Bulk density $\left(\mathrm{Mg} \mathrm{m}^{-3}\right)$ & 1.16 & NA \\
\hline Total organic carbon $(\%)$ & 1.43 & $0.57-2.51$ \\
\hline Organic matter $(\%)$ & 2.47 & NA \\
\hline Total N $(\%)$ & 0.08 & $0.04-0.17$ \\
\hline Exchangeable $\mathrm{NH}_{4}^{+}\left(\mathrm{mg} \mathrm{kg}^{-1}\right)$ & 21.02 & NA \\
\hline Available $\mathrm{NO}_{3}^{-}\left(\mathrm{mg} \mathrm{kg}^{-1}\right)$ & 7.01 & NA \\
\hline Available P (mg kg $\left.{ }^{-1}\right)$ & 4.85 & NA \\
\hline \multicolumn{3}{|c|}{ ( } \\
\hline Cation exchange capacity & 11.97 & $3.86-8.46$ \\
\hline Exchangeable $\mathrm{K}^{+}$ & 0.10 & $0.05-0.19$ \\
\hline Exchangeable $\mathrm{Ca}^{2+}$ & 0.25 & NA \\
\hline Exchangeable $\mathrm{Mg}^{2+}$ & 0.34 & NA \\
\hline Exchangeable $\mathrm{Na}^{+}$ & 0.22 & NA \\
\hline Exchangeable $\mathrm{Fe}^{2+}$ & 0.19 & NA \\
\hline Exchangeable $\mathrm{Cu}^{2+}$ & Trace & NA \\
\hline Exchangeable $\mathrm{Zn}^{2+}$ & 0.01 & NA \\
\hline Exchangeable $\mathrm{Mn}^{2+}$ & 0.02 & NA \\
\hline Total titratable acidity & 0.86 & NA \\
\hline Exchangeable $\mathrm{H}^{+}$ & 0.22 & NA \\
\hline Exchangeable $\mathrm{Al}^{3+}$ & 0.64 & NA \\
\hline Sand $(\%)$ & 71.04 & $72-76$ \\
\hline Silt (\%) & 14.58 & $8-9$ \\
\hline Clay (\%) & 14.38 & $16-19$ \\
\hline Texture (USDA) & Sandy loam & Sandy loam \\
\hline
\end{tabular}

Note: The information in Table 2 are based on soil dry weight; NA indicates not available; ${ }^{*}$ range reported by Paramananthan [28].

Table 2. Chemical composition of clinoptilolite zeolite.

\begin{tabular}{cc}
\hline Property & Clinoptilolite Zeolite (\%) \\
\hline Total N & 0.22 \\
Total P & 0.01 \\
Total K & 0.37 \\
Total Ca & 0.67 \\
Total Mg & 0.10 \\
Total Na & 0.76 \\
Total Fe & 0.11 \\
Total Zn & 15 \\
Total Mn & 17 \\
Total Cu & 125 \\
\hline
\end{tabular}

Note: The information in Table 2 are based on soil dry weight.

\subsection{Clinoptilolite Zeolite Characterization}

The CZ was purchased from MB Plus Sdn Bhd, Johore, Malaysia after which it analyzed for total $\mathrm{N}$ [29], exchangeable $\mathrm{NH}_{4}{ }^{+}$and available $\mathrm{NO}_{3}{ }^{-}$[27], $\mathrm{pH}, \mathrm{CEC}$ [30], and exchangeable $\mathrm{K}, \mathrm{Ca}$, and $\mathrm{Mg}$ [30]. The results of these analyses are presented Table 2.

\subsection{Determination of Nitrogen, Phosphorus, and Potassium Adsorption and Desorption}

In total, three replications were used for the $\mathrm{N}, \mathrm{P}$, and $\mathrm{K}$ adsorption and desorption in this study. A $2 \mathrm{~g}$ soil was weighed into a $250 \mathrm{~mL}$ centrifuge bottle. Thereafter, 0 , 50, 100, 200, 300, and $400 \mathrm{mg} \mathrm{N} \mathrm{L}^{-1}$ ) N solutions prepared. These concentrations were prepared by dissolving ammonium chloride $\left(\mathrm{NH}_{4} \mathrm{Cl}\right)$ in $0.2 \mathrm{M} \mathrm{NaCl}$. Afterwards, a $20 \mathrm{~mL}$ of the isonormal $\mathrm{N}$ solution was added to the centrifuge bottles to obtain $0,500,1000$, 
2000, 3000, and $4000 \mu \mathrm{g}$ of added $\mathrm{N}$ sample ${ }^{-1}$. The isonormal solution was used to preserve constant ionic strength in the mixtures (adsorbent and solution). Additionally, the isonormal solution was used to provide competing ions for exchange sites [31]. To de-activate micro-organisms activity, two drops of toluene were added to the samples [32] after which they were equilibrated for $24 \mathrm{~h}$ at $180 \mathrm{rpm}$ on an orbital shaker, centrifuged at $10,000 \mathrm{rpm}$ for $15 \mathrm{~min}$, and $\mathrm{N}$ determined [30]. Nitrogen adsorbed per gram soil ( $\mu \mathrm{g} \mathrm{g}{ }^{-1}$ soil) was calculated as the difference between the initial amount of $\mathrm{N}$ added and the amount in the equilibrium solution. After $\mathrm{N}$ adsorption, the samples were washed using ethanol followed by centrifugation at 10,000 rpm for $15 \mathrm{~min}$ [30] to discard the ethanol. Thereafter, a $20 \mathrm{~mL}$ of $2 \mathrm{M} \mathrm{KCl}$ was added, equilibrated for $24 \mathrm{~h}$ at $180 \mathrm{rpm}$ on an orbital shaker followed by centrifugation at 10,000 rpm for $15 \mathrm{~min}$. Afterwards, the desorbed $\mathrm{N}$ in the supernatant was determined [30].

The $\mathrm{N}$ adsorption procedure was used for the $\mathrm{P}$ adsorption but with some modifications. Phosphorus concentrations of 0, 50, 100, 200, 300, and $400 \mathrm{mg} \mathrm{P} \mathrm{L}^{-1}$ were prepared by dissolving potassium dihydrogen phosphate $\left(\mathrm{KH}_{2} \mathrm{PO}_{4}\right)$ in a $0.01 \mathrm{M} \mathrm{CaCl}_{2}$ solution, after which a $25 \mathrm{~mL}$ of the isonormal $\mathrm{P}$ solutions were added to the centrifuge bottles to obtain $0,652,1250,2500,3750$, and $5000 \mu \mathrm{g}$ of added $\mathrm{P}$ sample ${ }^{-1}$. Following centrifugation at $10,000 \mathrm{rpm}$ for $15 \mathrm{~min}, \mathrm{P}$ in the supernatant was determined [26]. For the amount of $\mathrm{P}$ desorbed, the centrifuged samples were used where a $20 \mathrm{~mL}$ of $0.01 \mathrm{M} \mathrm{CaCl}_{2}$ was added to the samples and equilibrated for $24 \mathrm{~h}$ at $180 \mathrm{rpm}$ on an orbital shaker, centrifuged at $10,000 \mathrm{rpm}$ for $15 \mathrm{~min}$, and the P in the supernatants was determined [26] as desorbed P.

The $\mathrm{N}$ adsorption procedure was used for the $\mathrm{K}$ adsorption but with some modifications. Potassium concentrations of $0,50,100,200,300$, and $400 \mathrm{mg} \mathrm{K} \mathrm{L}^{-1}$ were prepared by dissolving potassium chloride $(\mathrm{KCl})$ in $0.01 \mathrm{M} \mathrm{CaCl}_{2}$ solution after which, a $20 \mathrm{~mL}$ of the isonormal $\mathrm{K}$ solution was added to the centrifuge bottles to give $0,500,1000,2000,3000$, and $4000 \mu \mathrm{g}$ of added $\mathrm{K}$ sample ${ }^{-1}$, centrifuged at 10,000 rpm for $15 \mathrm{~min}$ after which the $\mathrm{K}$ in the supernatants were determined using atomic absorption spectrophotometery (AAS). For the amount of $\mathrm{K}$ desorbed, the centrifuged samples were used where a $20 \mathrm{~mL}$ of $0.01 \mathrm{M}$ $\mathrm{CaCl}_{2}$ was added to the samples and equilibrated for $24 \mathrm{~h}$ at $180 \mathrm{rpm}$ on an orbital shaker, centrifuged at 10,000 rpm for $15 \mathrm{~min}$, and the $\mathrm{K}$ in the supernatants determined using AAS as desorbed $\mathrm{K}$.

\subsection{Nitrogen, Phosphorus, and Potassium Adsorption Isotherms}

Adsorbed N, P, and $\mathrm{K}$ of the samples tested in this study were fitted to Langmuir type 1, 2, 3, and 4, Freundlich, and Temkin adsorption isotherms as shown in Table 3 [32,33].

Table 3. Nonlinear and linear adsorption isotherms models used in this present study.

\begin{tabular}{|c|c|c|c|c|}
\hline Isotherm & Nonlinear Form & Linear Form & Plot & Variables \\
\hline Langmuir -1 & \multirow{4}{*}{$\mathrm{q}_{\mathrm{e}}=\frac{\mathrm{q}_{\mathrm{m}} \mathrm{K}_{\mathrm{L}} \mathrm{C}_{\mathrm{e}}}{1+\mathrm{K}_{\mathrm{L}} \mathrm{C}_{\mathrm{e}}}$} & $\frac{\mathrm{C}_{\mathrm{e}}}{\mathrm{q}_{\mathrm{e}}}=\frac{\mathrm{C}_{\mathrm{e}}}{\mathrm{q}_{\mathrm{m}}}+\frac{1}{\mathrm{~K}_{\mathrm{L}} \mathrm{q}_{\mathrm{m}}}$ & $\frac{C_{e}}{q_{e}}$ vs $C_{e}$ & $\begin{array}{l}\mathrm{K}_{\mathrm{L}}=\frac{\text { slope }}{\text { intercept }} \\
\mathrm{q}_{\mathrm{m}}=\text { slope }^{-1}\end{array}$ \\
\hline Langmuir-2 & & $\frac{1}{\mathrm{q}_{\mathrm{e}}}=\left(\frac{1}{\mathrm{~K}_{\mathrm{L}} \mathrm{q}_{\mathrm{m}}}\right) \frac{1}{\mathrm{C}_{\mathrm{e}}}+\frac{1}{\mathrm{q}_{\mathrm{m}}}$ & $\frac{1}{\mathrm{q}_{\mathrm{e}}}$ vs $\frac{1}{\mathrm{C}_{\mathrm{e}}}$ & $\begin{array}{l}\mathrm{K}_{\mathrm{L}}=\frac{\text { intercept }}{\text { slope }} \\
\mathrm{q}_{\mathrm{m}}=\text { intercept }^{-1}\end{array}$ \\
\hline Langmuir -3 & & $\mathrm{q}_{\mathrm{e}}=\mathrm{q}_{\mathrm{m}}-\left(\frac{1}{\mathrm{~K}_{\mathrm{L}}}\right) \frac{\mathrm{q}_{\mathrm{e}}}{\mathrm{C}_{\mathrm{e}}}$ & $\mathrm{q}_{\mathrm{e}}$ vs $\frac{\mathrm{q}_{\mathrm{e}}}{\mathrm{C}_{\mathrm{e}}}$ & $\begin{array}{l}\mathrm{K}_{\mathrm{L}}=- \text { slope }^{-1} \\
\mathrm{q}_{\mathrm{m}}=\text { intercept }\end{array}$ \\
\hline Langmuir -4 & & $\frac{\mathrm{q}_{\mathrm{e}}}{\mathrm{C}_{\mathrm{e}}}=\mathrm{K}_{\mathrm{L}} \mathrm{q}_{\mathrm{m}}-\mathrm{K}_{\mathrm{L}} \mathrm{q}_{\mathrm{e}}$ & $\frac{\mathrm{q}_{\mathrm{e}}}{\mathrm{C}_{\mathrm{e}}}$ vs $\mathrm{q}_{\mathrm{e}}$ & $\begin{array}{l}\mathrm{K}_{\mathrm{L}}=- \text { slope } \\
\mathrm{q}_{\mathrm{m}}=-\frac{\text { intercept }}{\text { slope }}\end{array}$ \\
\hline Freundlich & $\mathrm{q}_{\mathrm{e}}=\mathrm{K}_{\mathrm{F}} \mathrm{C}_{\mathrm{e}}^{\frac{1}{n}}$ & $\begin{array}{l}\log \left(\mathrm{q}_{\mathrm{e}}\right)=\log \left(\mathrm{K}_{\mathrm{F}}\right)+ \\
\frac{1}{\mathrm{n}} \log \left(\mathrm{C}_{\mathrm{e}}\right)\end{array}$ & $\log \left(\mathrm{q}_{\mathrm{e}}\right)$ vs $\log \left(\mathrm{C}_{\mathrm{e}}\right)$ & $\begin{array}{l}\mathrm{K}_{\mathrm{F}}=\operatorname{antilog}(\text { intercept }) \\
\frac{1}{n}=\text { slope }\end{array}$ \\
\hline Temkin & $\mathrm{q}_{\mathrm{e}}=\mathrm{B}_{\mathrm{T}} \ln \left(\mathrm{K}_{\mathrm{T}} \mathrm{C}_{\mathrm{e}}\right)$ & $\mathrm{q}_{\mathrm{e}}=\mathrm{B}_{\mathrm{T}} \ln \mathrm{K}_{\mathrm{T}}+\mathrm{B}_{\mathrm{T}} \ln \mathrm{C}_{\mathrm{e}}$ & $\mathrm{q}_{\mathrm{e}} \mathrm{vs} \ln \mathrm{C}_{\mathrm{e}}$ & $\begin{array}{l}\mathrm{K}_{\mathrm{T}}=\exp \left(\frac{\text { intercept }}{\mathrm{B}_{\mathrm{T}}}\right) \\
\mathrm{B}_{\mathrm{T}}=\text { slope }\end{array}$ \\
\hline
\end{tabular}




\subsection{Soil pH Buffering Capacity Determination}

The different amounts of $\mathrm{CZ}$ and $250 \mathrm{~g}$ soil were thoroughly mixed as presented as follows:

$$
\begin{gathered}
\text { Soil: } 250 \text { g soil only } \\
\text { CZ: } 20 \text { g CZ only } \\
\text { CZ1: } 250 \text { g soil }+20 \text { g CZ } \\
\text { CZ2: } 250 \text { g soil }+40 \text { g CZ } \\
\text { CZ3: } 250 \text { g soil }+60 \text { g CZ }
\end{gathered}
$$

There were three replications in this soil $\mathrm{pH}$ buffering capacity study. The titration method was used to determine the soil and $\mathrm{CZ}$ pH buffering capacity [34] followed by plotting titration curves by increasing $\mathrm{mmol} \mathrm{H}^{+} \mathrm{kg}^{-1}$ samples suspended in water. A 1:2.5 sample:water (w:v) ratio was used for soil alone, CZ, CZ1, CZ2, and CZ3. During the titration, $10 \mathrm{~g}$ of soil, CZ, CZ1, CZ2, and CZ3 were weighed into $100 \mathrm{~mL}$ plastic vials followed by adding $25 \mathrm{~mL}$ distilled water. Then, $1 \mathrm{~mL} 0.05 \mathrm{M} \mathrm{CaCl}_{2}$ was added to the samples to minimize variations in ionic strength followed by adding $0.2 \mathrm{~mL}$ toluene to impede microbial activity [35]. Afterwards, the samples were equilibrated for $15 \mathrm{~min}$ at $180 \mathrm{rpm}$ on an orbital shaker for seven days at $25^{\circ} \mathrm{C}$ [35-37] after which a digital $\mathrm{pH}$ meter (SevenEasy $\mathrm{pH}$, Mettler-Toledo $\mathrm{GmbH}$, Switzerland) was used to determine the $\mathrm{pH}$ of the samples. For the samples, whose initial $\mathrm{pH}$ were less than 5.5, a $0.1 \mathrm{M} \mathrm{NaOH}$ was added using Eppendorf pipette to reduce suspension effect [34]. A $0.1 \mathrm{M} \mathrm{HCl}$ was used for the samples whose initial $\mathrm{pH}$ were greater six or were slightly acidic to basic. For all of the samples, $1,2,3,4,6,8$, and $10 \mathrm{~mL}$ of $0.1 \mathrm{M} \mathrm{HCl}$ or $0.1 \mathrm{M} \mathrm{NaOH}$ were used. The suspensions were stirred using a glass rod for $10 \mathrm{~s}$ following addition of $0.1 \mathrm{M} \mathrm{HCl}$ or $0.1 \mathrm{M}$ $\mathrm{NaOH}$. Thereafter, the $\mathrm{pH}$ of the suspensions were determined using a digital $\mathrm{pH}$ meter. The amount of $\mathrm{mmol} \mathrm{H}^{+}$needed to change $\mathrm{pH}$ by one unit was calculated as the negative reciprocal of the slope of the linear regression based on sample $\mathrm{pH}$ (Y-axis) and addition rate of $\mathrm{mmol} \mathrm{H}^{+} \mathrm{kg}^{-1}$ sample (X-axis):

$$
\text { Sample } \mathrm{pH} \text { buffering capacity }\left(\mathrm{mmol} \mathrm{H}{ }^{+} \mathrm{kg}^{-1} \text { sample }\right)=-\frac{1}{\text { slope }}
$$

where, slope $=$ fitted slope of linear regression line for each sample.

\subsection{Experimental Design and Statistical Analysis}

The treatments were arranged in a completely randomized design (CRD) with three replications and their effects were detected using analysis of variance followed by treatment means separation using Tukey's Test at $p \leq 0.05$. A simple linear regression was used to obtain the coefficient of determination $\left(R^{2}\right)$. These statistical tests were carried out using the Statistical Analysis System version 9.2 [38]. The N, P, and K adsorption isotherm equations were subjected to Chi-square analysis to obtain best-fit isotherm. The isotherm model with the smallest chi-square value was deemed the best best-fit isotherm. The stated formula was used for the Chi-square value calculation:

$$
x^{2}=\sum \frac{\left(\mathrm{q}_{\mathrm{e}}-\mathrm{q}_{\mathrm{e}, \mathrm{m}}\right)^{2}}{\mathrm{q}_{\mathrm{e}, \mathrm{m}}}
$$

where, $\mathrm{q}_{\mathrm{e}}$ is the equilibrium capacity from the experimental data and $\mathrm{q}_{\mathrm{e}, \mathrm{m}}$ is the equilibrium capacity obtained by calculation from model. 


\section{Results}

\subsection{Nutrient Concentrations in Equilibrated Samples}

The treatments and added N, P, and K interacted (Tables 4-6). Irrespective of treatment, the concentrations of $\mathrm{N}, \mathrm{P}$, and $\mathrm{K}$ in the equilibrated samples increased as the amounts of $\mathrm{N}$, P, and K increased (Tables 4-6). For CZ only, lower N and K was observed. Increasing rate of the $\mathrm{CZ}$ decreased $\mathrm{N}$ and $\mathrm{K}$ remaining in the equilibrated solution, however, it increased the remaining $P$ in the equilibrated solution (Tables 4-6). Regardless of the amount of $\mathrm{P}$ used, $\mathrm{P}$ in the equilibrated samples were similar (Table 5). Nitrogen and $\mathrm{K}$ in the equilibrated samples decreased with the increasing amount of the $C Z$ (Tables 4 and 6).

Table 4. Interactive effects of the different concentrations of nitrogen and treatments (Soil only, clinoptilolite zeolite only, and soil with different amounts of clinoptilolite zeolite) on nitrogen content.

\begin{tabular}{|c|c|c|c|c|c|c|}
\hline \multirow{3}{*}{ Treatment } & \multicolumn{6}{|c|}{ Nitrogen Left in the Equilibrated Samples $\left(\mu \mathrm{g} \mathrm{mL}^{-1}\right) C_{e}$} \\
\hline & 0 & 500 & 1000 & 2000 & 3000 & 4000 \\
\hline & \multicolumn{6}{|c|}{ Added N $(\mu \mathrm{g})$} \\
\hline Soil & $\begin{array}{l}23.35 \mathrm{~F} \mathrm{a} \\
( \pm 2.14)\end{array}$ & $\begin{array}{l}62.11 \mathrm{E} \mathrm{a} \\
( \pm 1.62)\end{array}$ & $\begin{array}{l}95.74 \mathrm{D} \mathrm{a} \\
( \pm 1.62)\end{array}$ & $\begin{array}{l}174.19 \mathrm{C} \mathrm{a} \\
( \pm 0.81)\end{array}$ & $\begin{array}{l}240.04 \mathrm{~B} \mathrm{a} \\
( \pm 2.14)\end{array}$ & $\begin{array}{l}294.21 \mathrm{~A} \mathrm{~b} \\
( \pm 1.40)\end{array}$ \\
\hline $\mathrm{CZ}$ & $\begin{array}{l}6.54 \mathrm{~F} \mathrm{c} \\
( \pm 0.81)\end{array}$ & $\begin{array}{l}14.94 \mathrm{E} \mathrm{e} \\
( \pm 1.62)\end{array}$ & $\begin{array}{l}28.95 \mathrm{D} \mathrm{d} \\
( \pm 1.62)\end{array}$ & $\begin{array}{l}50.44 \mathrm{Cd} \\
(2.81)\end{array}$ & $\begin{array}{l}77.99 \mathrm{~B} \mathrm{e} \\
( \pm 2.14)\end{array}$ & $\begin{array}{l}107.88 \mathrm{~A} \mathrm{e} \\
( \pm 1.40)\end{array}$ \\
\hline CZ1 & $\begin{array}{l}24.75 \mathrm{~F} \mathrm{a} \\
( \pm 1.62)\end{array}$ & $\begin{array}{l}49.51 \mathrm{E} \mathrm{b} \\
(0.81)\end{array}$ & $\begin{array}{l}84.06 \mathrm{D} \mathrm{b} \\
( \pm 1.40)\end{array}$ & $\begin{array}{l}139.17 \mathrm{C} \mathrm{b} \\
( \pm 1.62)\end{array}$ & $\begin{array}{l}212.02 \mathrm{~B} \mathrm{~b} \\
( \pm 1.62)\end{array}$ & $\begin{array}{l}298.41 \mathrm{~A} \mathrm{a} \\
( \pm 1.40)\end{array}$ \\
\hline $\mathrm{CZ2}$ & $\begin{array}{l}19.15 \mathrm{~F} \mathrm{~b} \\
( \pm 1.62)\end{array}$ & $\begin{array}{l}44.36 \mathrm{E} \mathrm{c} \\
( \pm 2.14)\end{array}$ & $\begin{array}{l}70.98 \mathrm{D} \mathrm{c} \\
( \pm 0.81)\end{array}$ & $\begin{array}{l}135.43 \mathrm{Cb} \\
( \pm 0.81)\end{array}$ & $\begin{array}{l}205.48 \text { B C } \\
( \pm 0.81)\end{array}$ & $\begin{array}{l}270.86 \text { A c } \\
( \pm 1.62)\end{array}$ \\
\hline $\mathrm{CZ3}$ & $\begin{array}{l}16.34 \mathrm{~F} \mathrm{~b} \\
( \pm 0.81)\end{array}$ & $\begin{array}{l}40.16 \mathrm{E} \mathrm{d} \\
( \pm 0.81)\end{array}$ & $\begin{array}{l}71.45 \mathrm{D} \mathrm{c} \\
( \pm 1.40)\end{array}$ & $\begin{array}{l}126.56 \mathrm{C} \mathrm{c} \\
( \pm 0.81)\end{array}$ & $\begin{array}{l}177.46 \mathrm{~B} \mathrm{~d} \\
( \pm 1.62)\end{array}$ & $\begin{array}{l}248.45 \mathrm{Ad} \\
( \pm 0.81)\end{array}$ \\
\hline
\end{tabular}

Note: Treatments (Soil only, clinoptilolite zeolite only, and soil with different amounts of clinoptilolite zeolite) and different nitrogen concentrations interacted at $p<0.0001$. Capital letters in a row and small letters in a column indicate significant difference (Tukey test) at $p \leq 0.05$.

Table 5. Interactive effects of the different concentrations of phosphorus and treatments (Soil only, clinoptilolite zeolite only, clinoptilolite zeolite only, and soil with different amounts of clinoptilolite zeolite) on phosphorus content.

\begin{tabular}{|c|c|c|c|c|c|c|}
\hline \multirow{3}{*}{ Treatment } & \multicolumn{6}{|c|}{ Phosphorus Left in the Equilibrated Samples $\left(\mu \mathrm{g} \mathrm{mL}^{-1}\right) \mathrm{C}_{e}$} \\
\hline & $\mathbf{0}$ & 675 & 1250 & 2500 & 3750 & 5000 \\
\hline & \multicolumn{6}{|c|}{ Added P ( $\mu g)$} \\
\hline Soil & $\begin{array}{l}0.06 \mathrm{~F} \mathrm{a} \\
( \pm 0.03)\end{array}$ & $\begin{array}{l}16.76 \text { E c } \\
( \pm 1.26)\end{array}$ & $\begin{array}{l}55.10 \mathrm{D} \mathrm{c} \\
( \pm 1.34)\end{array}$ & $\begin{array}{l}147.46 \mathrm{C} \mathrm{b} \\
( \pm 1.13)\end{array}$ & $\begin{array}{l}236.17 \mathrm{~B} \mathrm{~b} \\
( \pm 2.86)\end{array}$ & $\begin{array}{l}333.38 \mathrm{~A} \mathrm{~b} \\
( \pm 6.22)\end{array}$ \\
\hline $\mathrm{CZ}$ & $\begin{array}{l}0.02 \mathrm{~F} \mathrm{~b} \\
( \pm 0.00)\end{array}$ & $\begin{array}{l}41.03 \mathrm{E} \mathrm{a} \\
( \pm 0.95)\end{array}$ & $\begin{array}{l}89.00 \mathrm{D} \text { a } \\
( \pm 2.87)\end{array}$ & $\begin{array}{l}174.29 \mathrm{C} \mathrm{a} \\
( \pm 2.24)\end{array}$ & $\begin{array}{l}269.67 \mathrm{~B} \mathrm{a} \\
( \pm 5.27)\end{array}$ & $\begin{array}{l}365.13 \mathrm{~A} \mathrm{a} \\
( \pm 7.45)\end{array}$ \\
\hline CZ1 & $\begin{array}{l}0.02 \mathrm{~F} \mathrm{~b} \\
( \pm 0.01)\end{array}$ & $\begin{array}{l}18.50 \mathrm{E} \mathrm{bc} \\
( \pm 0.25)\end{array}$ & $\begin{array}{l}58.32 \mathrm{D} \text { bc } \\
( \pm 1.55)\end{array}$ & $\begin{array}{l}148.69 \mathrm{Cb} \\
( \pm 1.48)\end{array}$ & $\begin{array}{l}239.13 \mathrm{~B} \mathrm{~b} \\
( \pm 5.00)\end{array}$ & $\begin{array}{l}334.88 \mathrm{~A} \mathrm{~b} \\
( \pm 4.71)\end{array}$ \\
\hline $\mathrm{CZ2}$ & $\begin{array}{l}0.04 \mathrm{~F} \mathrm{ab} \\
( \pm 0.01)\end{array}$ & $\begin{array}{l}18.95 \mathrm{E} \mathrm{b} \\
( \pm 0.30)\end{array}$ & $\begin{array}{l}58.59 \mathrm{D} \text { bc } \\
( \pm 1.80)\end{array}$ & $\begin{array}{l}147.88 \mathrm{Cb} \\
( \pm 1.80)\end{array}$ & $\begin{array}{l}243.75 \mathrm{~B} \mathrm{~b} \\
( \pm 1.96)\end{array}$ & $\begin{array}{l}339.80 \mathrm{~A} \mathrm{~b} \\
( \pm 1.51)\end{array}$ \\
\hline $\mathrm{CZ3}$ & $\begin{array}{l}0.02 \mathrm{~F} \mathrm{~b} \\
( \pm 0.00)\end{array}$ & $\begin{array}{l}19.87 \mathrm{E} \mathrm{b} \\
( \pm 0.73)\end{array}$ & $\begin{array}{l}\text { 60.81 D b } \\
( \pm 1.41)\end{array}$ & $\begin{array}{l}151.29 \mathrm{Cb} \\
( \pm 1.99)\end{array}$ & $\begin{array}{l}242.29 \mathrm{~B} \mathrm{~b} \\
( \pm 1.48)\end{array}$ & $\begin{array}{l}341.04 \mathrm{~A} \mathrm{~b} \\
( \pm 2.15)\end{array}$ \\
\hline
\end{tabular}

Note: Treatments (Soil only, clinoptilolite zeolite only, and soil with different amounts of clinoptilolite zeolite) and different potassium concentrations interacted at $p<0.0001$. Capital letters in a row and small letters in a column indicate significant difference (Tukey test) at $p \leq 0.05$. 
Table 6. Interactive effects of the different potassium concentrations and treatments (Soil only, clinoptilolite zeolite only, and soil with different amounts of clinoptilolite zeolite) on potassium content.

\begin{tabular}{|c|c|c|c|c|c|c|}
\hline \multirow{3}{*}{ Treatment } & \multicolumn{6}{|c|}{ Potassium Left in the Equilibrated Samples $\left(\mu \mathrm{g} \mathrm{mL}^{-1}\right) \mathrm{C}_{\mathrm{e}}$} \\
\hline & 0 & 500 & 1000 & 2000 & 3000 & 4000 \\
\hline & \multicolumn{6}{|c|}{ Added K ( $\mu g)$} \\
\hline Soil & $\begin{array}{l}3.92 \mathrm{~F} \mathrm{a} \\
( \pm 0.08)\end{array}$ & $\begin{array}{l}45.37 \mathrm{E} \mathrm{a} \\
( \pm 0.31)\end{array}$ & $\begin{array}{l}85.80 \mathrm{D} \text { a } \\
( \pm 1.23)\end{array}$ & $\begin{array}{l}109.20 \mathrm{C} \mathrm{a} \\
( \pm 0.30)\end{array}$ & $\begin{array}{l}268.87 \mathrm{~B} \text { a } \\
( \pm 1.33)\end{array}$ & $\begin{array}{l}362.80 \mathrm{~A} \mathrm{a} \\
( \pm 2.09)\end{array}$ \\
\hline $\mathrm{CZ}$ & $\begin{array}{l}3.14 \mathrm{~F} \mathrm{~b} \\
( \pm 0.08)\end{array}$ & $\begin{array}{l}4.80 \mathrm{E} \mathrm{e} \\
( \pm 0.10)\end{array}$ & $\begin{array}{l}6.67 \mathrm{D} \mathrm{e} \\
( \pm 0.08)\end{array}$ & $\begin{array}{l}8.53 \mathrm{Ce} \\
( \pm 0.18)\end{array}$ & $\begin{array}{l}20.47 \mathrm{~B} \text { e } \\
( \pm 0.25)\end{array}$ & $\begin{array}{l}29.10 \mathrm{~A} \mathrm{e} \\
( \pm 0.17)\end{array}$ \\
\hline CZ1 & $\begin{array}{l}3.30 \mathrm{~F} \mathrm{~b} \\
( \pm 0.23)\end{array}$ & $\begin{array}{l}18.70 \mathrm{E} \mathrm{b} \\
( \pm 0.30)\end{array}$ & $\begin{array}{l}40.80 \mathrm{D} b \\
( \pm 0.89)\end{array}$ & $\begin{array}{l}57.83 \mathrm{Cb} \\
( \pm 0.38)\end{array}$ & $\begin{array}{l}\text { 179.07 B b } \\
( \pm 1.10)\end{array}$ & $\begin{array}{l}262.47 \mathrm{~A} \mathrm{~b} \\
( \pm 1.33)\end{array}$ \\
\hline $\mathrm{CZ2}$ & $\begin{array}{l}3.12 \mathrm{~F} \mathrm{~b} \\
( \pm 0.08)\end{array}$ & $\begin{array}{l}12.67 \mathrm{E} \mathrm{c} \\
( \pm 0.15)\end{array}$ & $\begin{array}{l}26.87 \mathrm{D} \mathrm{c} \\
( \pm 0.38)\end{array}$ & $\begin{array}{l}37.00 \text { C c } \\
( \pm 0.17)\end{array}$ & $\begin{array}{l}\text { 133.67 B c } \\
( \pm 0.31)\end{array}$ & $\begin{array}{l}193.53 \mathrm{~A} \mathrm{c} \\
( \pm 1.53)\end{array}$ \\
\hline CZ3 & $\begin{array}{l}3.15 \mathrm{~F} \mathrm{~b} \\
( \pm 0.10)\end{array}$ & $\begin{array}{l}10.00 \mathrm{E} \mathrm{d} \\
( \pm 0.10)\end{array}$ & $\begin{array}{l}\text { 20.73 D d } \\
( \pm 0.15)\end{array}$ & $\begin{array}{l}26.97 \mathrm{Cd} \\
( \pm 0.38)\end{array}$ & $\begin{array}{l}101.60 \mathrm{~B} \mathrm{~d} \\
( \pm 0.53)\end{array}$ & $\begin{array}{l}149.93 \mathrm{Ad} \mathrm{d} \\
( \pm 0.76)\end{array}$ \\
\hline
\end{tabular}

Note: Treatments (Soil only, clinoptilolite zeolite only, and soil with different amounts of clinoptilolite zeolite) and amounts of $\mathrm{K}$ interacted at $p<0.0001$. Capital letters in a row and small letters in a column indicate significant difference (Tukey test) at $p \leq 0.05$.

Irrespective of treatment (soil alone, $\mathrm{CZ}$ alone, CZ1, CZ2, and CZ3), there was a linear relationship between the added amounts of $\mathrm{N}, \mathrm{P}$, and $\mathrm{K}$ and the contents in the equilibrated samples (Table 7), suggesting that $\mathrm{N}, \mathrm{P}$, and $\mathrm{K}$ contents in the equilibrated samples increased with the increasing amounts of N, P, and K used.

Table 7. Linear relationships between the added amounts nitrogen, phosphorus, and potassium and their contents in the equilibrated samples.

\begin{tabular}{|c|c|c|}
\hline Treatment & Regression Equation & $\mathbf{R}^{2}$ Value \\
\hline \multicolumn{3}{|c|}{ 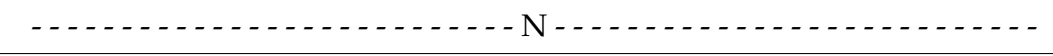 } \\
\hline Soil & $y=28.128+0.067 x$ & $0.9954 * *$ \\
\hline $\mathrm{CZ}$ & $y=3.435+0.025 x$ & $0.9941^{* *}$ \\
\hline CZ1 & $y=16.314+0.068 x$ & $0.9906^{* *}$ \\
\hline CZ2 & $y=12.681+0.064 x$ & $0.9973 * *$ \\
\hline CZ3 & $y=13.183+0.057 x$ & $0.9965^{* *}$ \\
\hline \multicolumn{3}{|c|}{ - - - - - - - - - - - - - - - - - - - - P - - - - - - - - - - - - - - - - - - - - } \\
\hline Soil & $y=-19.377+0.069 x$ & $0.9902 * *$ \\
\hline CZ & $y=-3.877+0.073 x$ & $0.9993 * *$ \\
\hline $\mathrm{CZ1}$ & $y=-18.033+0.069 x$ & $0.9917^{* *}$ \\
\hline CZ2 & $y=-18.798+0.070 x$ & $0.9910 * *$ \\
\hline CZ3 & $y=-17.605+0.070 x$ & $0.9921^{* *}$ \\
\hline \multicolumn{3}{|c|}{ 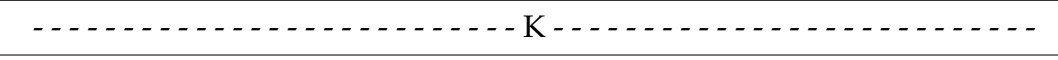 } \\
\hline Soil & $y=-9.004+0.089 x$ & $0.9456^{* *}$ \\
\hline $\mathrm{CZ}$ & $y=0.811+0.006 x$ & $0.9079 * *$ \\
\hline CZ1 & $y=-19.646+0.065 x$ & $0.9105^{* *}$ \\
\hline $\mathrm{CZ2}$ & $y=-16.243+0.048 x$ & $0.8916^{* *}$ \\
\hline CZ3 & $y=-12.414+0.037 x$ & $0.8817^{* *}$ \\
\hline
\end{tabular}

Note: Two ${ }^{* *}$ indicate significant relationship at $p=0.01$.

\subsection{Adsorption Isotherm of Nitrogen, Phosphorus, and Potassium}

Adsorption of $\mathrm{N}$ and $\mathrm{K}$ were higher in all treatments with $\mathrm{CZ}$ compared with soil alone (Tables 8-10). 
Table 8. Interactive effects of the different nitrogen concentrations and treatments (Soil only, clinoptilolite zeolite only, and soil with different amounts of clinoptilolite zeolite) on the amounts of nitrogen adsorbed.

\begin{tabular}{|c|c|c|c|c|c|}
\hline \multirow{3}{*}{ Treatment } & \multicolumn{5}{|c|}{ Adsorbed $N\left(\mu g^{-1}\right) q_{e}$} \\
\hline & 500 & 1000 & 2000 & 3000 & 4000 \\
\hline & \multicolumn{5}{|c|}{ Added N ( $\mu \mathrm{g})$} \\
\hline Soil & $\begin{array}{l}112.39 \mathrm{E} \mathrm{c} \\
( \pm 16.18)\end{array}$ & $\begin{array}{l}276.15 \mathrm{D} \mathrm{d} \\
( \pm 16.18)\end{array}$ & $\begin{array}{l}491.59 \mathrm{Cd} \\
( \pm 8.09)\end{array}$ & $\begin{array}{l}833.12 \mathrm{~B} \mathrm{~d} \\
( \pm 21.40)\end{array}$ & $\begin{array}{l}1291.40 \mathrm{~A} \mathrm{~d} \\
( \pm 14.01)\end{array}$ \\
\hline $\mathrm{CZ}$ & $\begin{array}{l}415.96 \mathrm{E} \mathrm{a} \\
( \pm 16.18)\end{array}$ & $\begin{array}{l}775.86 \mathrm{D} \text { a } \\
( \pm 16.18)\end{array}$ & $\begin{array}{l}1561.04 \mathrm{C} \mathrm{a} \\
( \pm 28.02)\end{array}$ & $\begin{array}{l}2285.51 \mathrm{~B} \mathrm{a} \\
( \pm 21.40)\end{array}$ & $\begin{array}{l}2986.63 \mathrm{~A} \mathrm{a} \\
( \pm 14.01)\end{array}$ \\
\hline CZ1 & $\begin{array}{l}252.48 \mathrm{E} \mathrm{b} \\
( \pm 8.09)\end{array}$ & $\begin{array}{l}406.90 \mathrm{D} \mathrm{c} \\
( \pm 14.01)\end{array}$ & $\begin{array}{l}855.84 \mathrm{C} \text { bc } \\
( \pm 16.18)\end{array}$ & $\begin{array}{l}\text { 1127.32 B c } \\
( \pm 16.18)\end{array}$ & $\begin{array}{l}\text { 1263.37 A d } \\
( \pm 14.01)\end{array}$ \\
\hline CZ2 & $\begin{array}{l}247.85 \mathrm{E} \mathrm{b} \\
( \pm 21.40)\end{array}$ & $\begin{array}{l}481.66 \mathrm{D} \mathrm{b} \\
( \pm 8.09)\end{array}$ & $\begin{array}{l}837.20 \mathrm{C} \mathrm{c} \\
( \pm 8.09)\end{array}$ & $\begin{array}{l}1136.70 \text { B c } \\
( \pm 8.09)\end{array}$ & $\begin{array}{l}\text { 1482.90 A c } \\
( \pm 16.18)\end{array}$ \\
\hline CZ3 & $\begin{array}{l}261.88 \mathrm{E} \mathrm{b} \\
( \pm 8.09)\end{array}$ & $\begin{array}{l}448.99 \mathrm{D} \mathrm{b} \\
( \pm 14.01)\end{array}$ & $\begin{array}{l}897.93 \mathrm{Cb} \\
( \pm 8.09)\end{array}$ & $\begin{array}{l}1388.90 \mathrm{~B} \mathrm{~b} \\
( \pm 16.18)\end{array}$ & $\begin{array}{l}1679.06 \text { A b } \\
( \pm 8.09)\end{array}$ \\
\hline
\end{tabular}

Note: Treatments (Soil only, clinoptilolite zeolite only, and soil with different amounts of clinoptilolite zeolite) and amounts of $\mathrm{N}$ interacted at $p<0.0001$. Capital letters in a row and small letters in a column indicate significant difference (Tukey test) at $p \leq 0.05$.

Table 9. Interactive effects of the different phosphorus concentrations and treatments (Soil only, clinoptilolite zeolite only, and soil with different amounts of clinoptilolite zeolite) on the amounts of phosphorus adsorbed.

\begin{tabular}{|c|c|c|c|c|c|}
\hline \multirow{3}{*}{ Treatment } & \multicolumn{5}{|c|}{ Adsorbed Phosphorus $\left(\mu g g^{-1}\right) q_{e}$} \\
\hline & 625 & 1250 & 2500 & 3750 & 5000 \\
\hline & \multicolumn{5}{|c|}{ Added P ( $\mu g)$} \\
\hline Soil & $\begin{array}{l}416.31 \mathrm{C} \mathrm{a} \\
( \pm 15.79)\end{array}$ & $\begin{array}{l}562.00 \mathrm{~B} \mathrm{a} \\
( \pm 16.81)\end{array}$ & $\begin{array}{l}675.52 \mathrm{~B} \mathrm{a} \\
( \pm 14.18)\end{array}$ & $\begin{array}{l}798.68 \mathrm{~A} \mathrm{a} \\
( \pm 35.78)\end{array}$ & $\begin{array}{l}833.56 \mathrm{~A} \text { a } \\
( \pm 77.75)\end{array}$ \\
\hline $\mathrm{CZ}$ & $\begin{array}{l}112.40 \mathrm{~B} \mathrm{c} \\
( \pm 11.86)\end{array}$ & $\begin{array}{l}137.75 \mathrm{~B} \mathrm{c} \\
( \pm 35.86)\end{array}$ & $\begin{array}{l}321.61 \mathrm{~A} \mathrm{~b} \\
( \pm 27.96)\end{array}$ & $\begin{array}{l}379.42 \mathrm{~A} \mathrm{~b} \\
( \pm 65.85)\end{array}$ & $\begin{array}{l}436.19 \mathrm{~A} \mathrm{~b} \\
( \pm 93.08)\end{array}$ \\
\hline CZ1 & $\begin{array}{l}394.00 \mathrm{D} \mathrm{ab} \\
( \pm 3.15)\end{array}$ & $\begin{array}{l}521.22 \mathrm{C} \mathrm{ab} \\
( \pm 19.42)\end{array}$ & $\begin{array}{l}641.66 \mathrm{~B} \mathrm{a} \\
( \pm 18.47)\end{array}$ & $\begin{array}{l}761.19 \mathrm{~A} \mathrm{a} \\
( \pm 62.56)\end{array}$ & $\begin{array}{l}814.32 \mathrm{~A} \mathrm{a} \\
( \pm 58.94)\end{array}$ \\
\hline $\mathrm{CZ2}$ & $\begin{array}{l}388.63 \mathrm{D} \mathrm{b} \\
( \pm 3.79)\end{array}$ & $\begin{array}{l}518.14 \mathrm{C} \text { ab } \\
( \pm 22.47)\end{array}$ & $\begin{array}{l}652.06 \mathrm{~B} \mathrm{a} \\
( \pm 22.49)\end{array}$ & $\begin{array}{l}703.63 \mathrm{AB} \text { a } \\
( \pm 24.56)\end{array}$ & $\begin{array}{l}753.10 \mathrm{~A} \mathrm{a} \\
( \pm 18.84)\end{array}$ \\
\hline $\mathrm{CZ3}$ & $\begin{array}{l}376.92 \mathrm{D} \mathrm{b} \\
( \pm 9.03)\end{array}$ & $\begin{array}{l}490.11 \mathrm{C} \mathrm{b} \\
( \pm 17.54)\end{array}$ & $\begin{array}{l}609.10 \mathrm{~B} \mathrm{a} \\
( \pm 24.83)\end{array}$ & $\begin{array}{l}721.60 \mathrm{~A} \mathrm{a} \\
( \pm 18.51)\end{array}$ & $\begin{array}{l}737.23 \mathrm{~A} \mathrm{a} \\
( \pm 26.90)\end{array}$ \\
\hline
\end{tabular}

Note: The treatments (Soil only, clinoptilolite zeolite only, and soil with different amounts of clinoptilolite zeolite) and amounts of $P$ interacted at $p<0.0001$. Capital letters in a row and small letters in a column indicate significant difference (Tukey test) at $p \leq 0.05$. 
Table 10. Interactive effects of the different potassium concentrations and treatments (Soil only, clinoptilolite zeolite only, and soil with different amounts of clinoptilolite zeolite) on the amounts of potassium adsorbed.

\begin{tabular}{|c|c|c|c|c|c|}
\hline \multirow{3}{*}{ Treatment } & \multicolumn{5}{|c|}{ Adsorbed Potassium $\left(\mu g g^{-1}\right) q_{e}$} \\
\hline & 500 & 1000 & 2000 & 3000 & 4000 \\
\hline & \multicolumn{5}{|c|}{ Added K ( $\mu \mathrm{g})$} \\
\hline Soil & $\begin{array}{l}85.53 \mathrm{E} \mathrm{e} \\
( \pm 3.06)\end{array}$ & $\begin{array}{l}181.20 \mathrm{De} \\
( \pm 12.29)\end{array}$ & $\begin{array}{l}947.20 \mathrm{~A} \mathrm{e} \\
( \pm 3.00)\end{array}$ & $\begin{array}{l}350.53 \mathrm{C} \text { e } \\
( \pm 13.32)\end{array}$ & $\begin{array}{l}411.20 \mathrm{~B} \mathrm{e} \\
( \pm 20.88)\end{array}$ \\
\hline $\mathrm{CZ}$ & $\begin{array}{l}483.30 \mathrm{E} \mathrm{a} \\
( \pm 1.00)\end{array}$ & $\begin{array}{l}964.63 \mathrm{D} \text { a } \\
( \pm 0.76)\end{array}$ & $\begin{array}{l}1945.97 \mathrm{C} \mathrm{a} \\
( \pm 1.76)\end{array}$ & $\begin{array}{l}2826.63 \mathrm{~B} \mathrm{a} \\
( \pm 2.52)\end{array}$ & $\begin{array}{l}3740.30 \mathrm{~A} \mathrm{a} \\
( \pm 1.73)\end{array}$ \\
\hline CZ1 & $\begin{array}{l}346.00 \mathrm{E} \mathrm{d} \\
( \pm 3.00)\end{array}$ & $\begin{array}{l}625.00 \mathrm{D} \mathrm{d} \\
( \pm 8.89)\end{array}$ & $\begin{array}{l}1454.67 \mathrm{~A} \mathrm{~d} \\
( \pm 3.79)\end{array}$ & $\begin{array}{l}1242.33 \mathrm{Cd} \\
( \pm 11.02)\end{array}$ & $\begin{array}{l}1408.33 \mathrm{~B} \mathrm{~d} \\
( \pm 13.32)\end{array}$ \\
\hline CZ2 & $\begin{array}{l}404.53 \text { E c } \\
( \pm 1.53)\end{array}$ & $\begin{array}{l}762.53 \mathrm{D} \mathrm{c} \\
( \pm 3.79)\end{array}$ & $\begin{array}{l}1661.20 \text { C c } \\
( \pm 1.73)\end{array}$ & $\begin{array}{l}1694.53 \mathrm{~B} \mathrm{c} \\
( \pm 3.06)\end{array}$ & $\begin{array}{l}\text { 2095.87 A c } \\
( \pm 15.28)\end{array}$ \\
\hline CZ3 & $\begin{array}{l}431.50 \mathrm{E} \mathrm{b} \\
( \pm 1.00)\end{array}$ & $\begin{array}{l}824.17 \mathrm{D} \mathrm{b} \\
( \pm 1.53)\end{array}$ & $\begin{array}{l}1761.83 \mathrm{Cb} \\
( \pm 3.79)\end{array}$ & $\begin{array}{l}2015.50 \mathrm{~B} \mathrm{~b} \\
( \pm 2.59)\end{array}$ & $\begin{array}{l}2532.17 \mathrm{~A} \mathrm{~b} \\
( \pm 7.57)\end{array}$ \\
\hline
\end{tabular}

Note: Treatments (Soil only, clinoptilolite zeolite only, and soil with different amounts of clinoptilolite zeolite) and amounts of $\mathrm{K}$ interacted at $p<0.0001$. Capital letters in a row and small letters in a column indicate significant difference (Tukey test) at $p \leq 0.05$.

Irrespective of the samples tested, there was an increase in adsorptions in the amounts of N, P, and $\mathrm{K}$ adsorbed (Table 11). The CZ showed higher $\mathrm{N}$ and $\mathrm{K}$ adsorption rates but lower $\mathrm{P}$ adsorption rate compared with soil alone (Table 11).

Table 11. Linear relationships between the added nitrogen, phosphorus, and potassium concentrations and the amounts of nitrogen, phosphorus, and potassium adsorbed.

\begin{tabular}{|c|c|c|}
\hline Treatment & Regression Equation & $\mathbf{R}^{2}$ Value \\
\hline \multicolumn{3}{|c|}{ 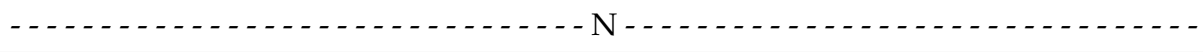 } \\
\hline Soil & $y=-83.035+0.326 x$ & $0.9746^{* *}$ \\
\hline $\mathrm{CZ}$ & $y=53.933+0.739 x$ & $0.9994 * *$ \\
\hline CZ1 & $y=146.621+0.302 x$ & $0.9507^{* *}$ \\
\hline $\mathrm{CZ2}$ & $y=112.365+0.345 x$ & $0.9954 * *$ \\
\hline $\mathrm{CZ3}$ & $y=54.958+0.419 x$ & $0.9915^{* *}$ \\
\hline \multicolumn{3}{|c|}{ 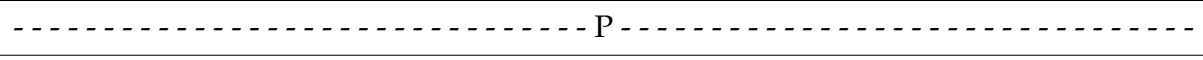 } \\
\hline Soil & $y=409.661+0.093 x$ & $0.9154^{* *}$ \\
\hline $\mathrm{CZ}$ & $y=70.878+0.079 x$ & $0.9219^{* *}$ \\
\hline CZ1 & $y=379.546+0.094 x$ & $0.9397 * *$ \\
\hline $\mathrm{CZ2}$ & $y=396.420+0.079 x$ & $0.8676^{* *}$ \\
\hline CZ3 & $y=370.058+0.083 x$ & $0.8987^{* *}$ \\
\hline \multicolumn{3}{|c|}{ 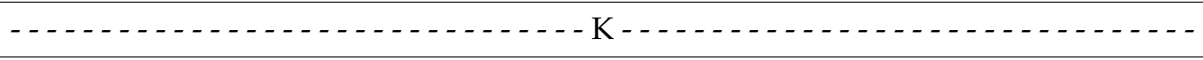 } \\
\hline Soil & $y=224.575+0.081 x$ & $0.1726^{\text {ns }}$ \\
\hline $\mathrm{CZ}$ & $y=40.336+0.929 x$ & $0.9994^{* *}$ \\
\hline $\mathrm{CZ1}$ & $y=398.747+0.294 x$ & $0.6096^{\mathrm{ns}}$ \\
\hline $\mathrm{CZ2}$ & $y=336.429+0.470 x$ & $0.8704 *$ \\
\hline $\mathrm{CZ3}$ & $y=270.473+0.592 x$ & $0.9438 * *$ \\
\hline
\end{tabular}

Note: Two ${ }^{* *}$ indicate significant relationship at $p=0.01$. One ${ }^{*}$ indicates significant relationship at $p=0.05 . \mathrm{ns}$ indicates not significant at $p=0.05$.

\subsection{Nitrogen, Phosphorus, and Potassium Adsorption Isotherms}

The intercepts for types 1, 2, and 3 of Langmuir $\mathrm{N}$ adsorption isotherms were negative (Table 12). Regardless of treatment, the $\mathrm{N}$ adsorption experimental data best fitted with Freundlich and this observation is related the significant $\mathrm{R}^{2}$ and lower $\chi^{2}$ value (Table 13). This is unlike those with Langmuir (type 4) and Temkin adsorption equations (Table 13). 
High antilog (intercept) $\mathrm{K}_{\mathrm{F}}$ values ( $\mathrm{N}$ adsorption) were observed in $\mathrm{CZ}$ alone, $\mathrm{CZ1}, \mathrm{CZ2}$, and CZ3 compared with soil alone (Table 14).

Table 12. Fitting Langmuir type 1, 2, and 3 isotherms to the nitrogen adsorption data based on simple regression and Chi-square analyses results.

\begin{tabular}{|c|c|c|c|}
\hline Treatment & Regression Equation & $\mathbf{R}^{2}$ & $x^{2}$ \\
\hline \multicolumn{4}{|c|}{ 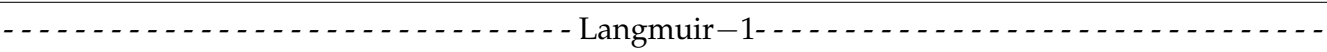 } \\
\hline Soil & $y=0.101-0.0002 x$ & 0.7476 * & $2.71 \times 10^{-3}$ \\
\hline $\mathrm{CZ}$ & $y=0.016-0.00001 x$ & $-0.0357^{\mathrm{ns}}$ & $1.82 \times 10^{-4}$ \\
\hline CZ1 & $y=0.062-0.00003 x$ & $-0.0035^{\mathrm{ns}}$ & $2.45 \times 10^{-3}$ \\
\hline CZ2 & $y=0.054-0.00002 x$ & $-0.0349 \mathrm{~ns}$ & $1.39 \times 10^{-3}$ \\
\hline CZ3 & $y=0.052-0.00005 x$ & $0.5247^{\mathrm{ns}}$ & $7.89 \times 10^{-4}$ \\
\hline \multicolumn{4}{|c|}{ 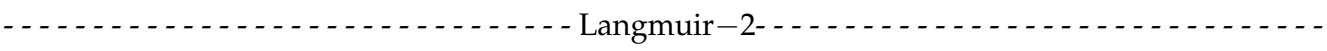 } \\
\hline Soil & $y=-0.00023+0.111 x$ & $0.9720 * *$ & $4.17 \times 10^{-5}$ \\
\hline $\mathrm{CZ}$ & $y=-0.00002+0.017 x$ & $0.9980 * *$ & $3.90 \times 10^{-5}$ \\
\hline CZ1 & $y=-0.00009+0.069 x$ & $0.9903 * *$ & $2.55 \times 10^{-5}$ \\
\hline $\mathrm{CZ2}$ & $y=-0.00007+0.060 x$ & $0.9782 * *$ & $2.66 \times 10^{-5}$ \\
\hline CZ3 & $y=-0.00007+0.055 x$ & 0.9980 ** & $6.75 \times 10^{-6}$ \\
\hline
\end{tabular}

Note: Two ${ }^{* *}$ indicate significant relationship at $p=0.01$. One ${ }^{*}$ indicates significant relationship at $p=0.05 . \mathrm{ns}$ indicates not significant at $p=0.05$.

Table 13. Fitting Langmuir type 4, Freundlich, and Temkin isotherms to the nitrogen adsorption data based on simple regression and Chi-square analyses results.

\begin{tabular}{|c|c|c|c|}
\hline Treatment & Regression Equation & $\mathbf{R}^{2}$ & $x^{2}$ \\
\hline \multicolumn{4}{|c|}{ - } \\
\hline Soil & $y=10.154+0.0015 x$ & 0.8848 * & 0.273 \\
\hline $\mathrm{CZ}$ & $y=62.131+0.0008 x$ & $-0.0013^{\mathrm{ns}}$ & 0.761 \\
\hline CZ1 & $y=16.109+0.0006 x$ & $0.0704^{\mathrm{ns}}$ & 0.696 \\
\hline $\mathrm{CZ2}$ & $y=18.565+0.0004 x$ & $-0.0224^{\mathrm{ns}}$ & 0.457 \\
\hline $\mathrm{CZ3}$ & $y=19.107+0.0009 x$ & $0.5794^{\text {ns }}$ & 0.291 \\
\hline \multicolumn{4}{|c|}{ 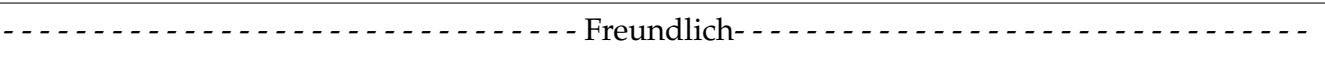 } \\
\hline Soil & $y=0.414+1.337 x$ & $0.9932 * *$ & $9.45 \times 10^{-4}$ \\
\hline $\mathrm{CZ}$ & $y=1.734+1.048 x$ & $0.9953^{* *}$ & $5.14 \times 10^{-4}$ \\
\hline $\mathrm{CZ1}$ & $y=1.019+1.109 x$ & $0.9832 * *$ & $1.77 \times 10^{-3}$ \\
\hline CZ2 & $y=1.141+1.074 x$ & $0.9896^{* *}$ & $1.22 \times 10^{-3}$ \\
\hline $\mathrm{CZ3}$ & $y=1.072+1.131 x$ & $0.9957^{* *}$ & $4.54 \times 10^{-4}$ \\
\hline \multicolumn{4}{|c|}{ 年 } \\
\hline Soil & $y=-11411+2820.63 x$ & $0.8989 * *$ & 870.84 \\
\hline $\mathrm{CZ}$ & $y=-7985+3071.78 x$ & $0.9302 * *$ & 1356.40 \\
\hline CZ1 & $y=-9620+2569.88 x$ & $0.9665^{* *}$ & 393.00 \\
\hline $\mathrm{CZ} 2$ & $y=-9153+2530.55 x$ & $0.9508^{* *}$ & 330.51 \\
\hline $\mathrm{CZ3}$ & $y=-9765+2723.95 x$ & $0.9320 * *$ & 951.02 \\
\hline \multicolumn{4}{|c|}{ ـ } \\
\hline Soil & $y=-5825.393+596.686 x$ & $0.8848^{*}$ & 5726 \\
\hline $\mathrm{CZ}$ & $y=-16184.00+305.264 x$ & $-0.0013^{\mathrm{ns}}$ & 5224 \\
\hline CZ1 & $y=-5783.607+483.720 x$ & $0.0704^{\mathrm{ns}}$ & 3549 \\
\hline $\mathrm{CZ2}$ & $y=-7957.093+549.928 x$ & $-0.0224^{\mathrm{ns}}$ & 3586 \\
\hline CZ3 & $y=-13020.00+731.534 x$ & $0.5794^{\text {ns }}$ & 1418 \\
\hline
\end{tabular}

Note: Two ${ }^{* *}$ indicate significant relationship at $p=0.01$. One ${ }^{*}$ indicates significant relationship at $p=0.05 . \mathrm{ns}$ indicates not significant at $p=0.05$. 
Table 14. Adsorption capacity (KF) and adsorption isotherm constant of Freundlich in relation to adsorption condition $(1 / n)$ for nitrogen adsorption.

\begin{tabular}{lll}
\hline \multirow{2}{*}{ Treatment } & Freundlich & \\
\cline { 2 - 3 } & $\begin{array}{l}\mathbf{K}_{\mathbf{F}} \\
\left(\boldsymbol{\mu g} \mathbf{g}^{-\mathbf{1}} \mathbf{)}\right.\end{array}$ & $\frac{1}{n}$ \\
\hline Soil & 2.594 & 1.337 \\
CZ & 54.200 & 1.048 \\
CZ1 & 10.447 & 1.109 \\
CZ2 & 13.836 & 1.074 \\
CZ3 & 11.803 & 1.131 \\
\hline
\end{tabular}

The $\mathrm{P}$ adsorption data for soil alone, CZ alone, CZ1, CZ2, and CZ3 best fitted with Langmuir type 2 isotherm, based on significant $R^{2}$ and lower $\chi^{2}$ values (Table 15) and this is in contrast to those of Freundlich and Temkin (Table 16). The treatment without CZ (Soil only) demonstrated the highest bonding energy constant $\left(\mathrm{K}_{\mathrm{L}}\right)$ for $\mathrm{P}$ adsorption (Table 17).

Table 15. Fitting Langmuir type 1,2, and 3 isotherms to the phosphorus adsorption data based on simple regression and Chi-square analyses results.

\begin{tabular}{|c|c|c|c|}
\hline Treatment & Regression Equation & $\mathbf{R}^{2}$ & $x^{2}$ \\
\hline \multicolumn{4}{|c|}{ ـ } \\
\hline Soil & $y=0.021+0.0001 x$ & $0.8371 *$ & $4.30 \times 10^{-3}$ \\
\hline $\mathrm{CZ}$ & $y=0.058+0.00003 x$ & $0.5481^{\mathrm{ns}}$ & $4.51 \times 10^{-4}$ \\
\hline CZ1 & $y=0.023+0.0001 x$ & $0.8315 *$ & $3.90 \times 10^{-3}$ \\
\hline $\mathrm{CZ2}$ & $y=0.023+0.0001 x$ & 0.8553 * & $3.46 \times 10^{-3}$ \\
\hline $\mathrm{CZ3}$ & $y=0.025+0.0001 x$ & $0.8347^{*}$ & $3.64 \times 10^{-3}$ \\
\hline \multicolumn{4}{|c|}{ 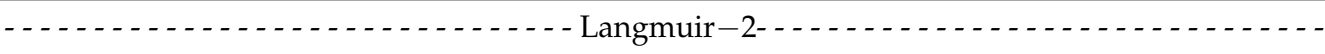 } \\
\hline Soil & $y=0.0002+0.013 x$ & $0.9362 * *$ & $5.63 \times 10^{-5}$ \\
\hline $\mathrm{CZ}$ & $y=0.0001+0.054 x$ & $0.9939 * *$ & $7.49 \times 10^{-6}$ \\
\hline CZ1 & $y=0.0002+0.015 x$ & $0.9410^{* *}$ & $5.36 \times 10^{-5}$ \\
\hline $\mathrm{CZ2}$ & $y=0.0002+0.016 x$ & $0.9467 * *$ & $4.81 \times 10^{-5}$ \\
\hline CZ3 & $y=0.0002+0.017 x$ & $0.9447^{* *}$ & $5.09 \times 10^{-5}$ \\
\hline \multicolumn{4}{|c|}{ 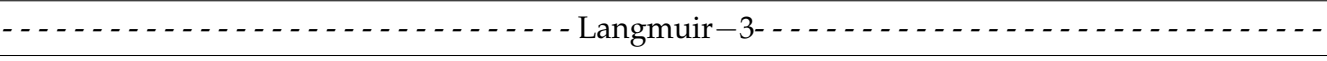 } \\
\hline Soil & $y=5948.693-87.142 x$ & $0.5825^{\mathrm{ns}}$ & 1685 \\
\hline CZ1 & $y=6219.856-103.925 x$ & $0.5878^{\mathrm{ns}}$ & 1729 \\
\hline $\mathrm{CZ2}$ & $y=6257.001-108.028 x$ & $0.6224^{\mathrm{ns}}$ & 1668 \\
\hline $\mathrm{CZ3}$ & $y=6364.248-116.858 x$ & $0.5996^{\mathrm{ns}}$ & 1750 \\
\hline
\end{tabular}

Note: Two ${ }^{* *}$ indicate significant relationship at $p=0.01$. One ${ }^{*}$ indicates significant relationship at $p=0.05 . \mathrm{ns}$ indicates not significant at $p=0.05$. 
Table 16. Fitting Langmuir type 4, Freundlich, and Temkin isotherms to the phosphorus adsorption data based on simple regression and Chi-square analyses results.

\begin{tabular}{|c|c|c|c|}
\hline Treatment & Regression Equation & $\mathbf{R}^{2}$ & $x^{2}$ \\
\hline \multicolumn{4}{|c|}{ 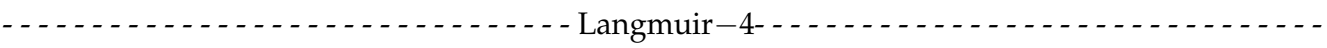 } \\
\hline Soil & $y=56.484-0.008 x$ & $0.5825^{\mathrm{ns}}$ & 14.057 \\
\hline $\mathrm{CZ}$ & $y=17.418-0.0005 x$ & $0.4944^{\mathrm{ns}}$ & 0.131 \\
\hline CZ1 & $y=50.176-0.007 x$ & $0.5878^{\mathrm{ns}}$ & 10.155 \\
\hline $\mathrm{CZ2}$ & $y=49.458-0.007 x$ & $0.6224^{\mathrm{ns}}$ & 9.032 \\
\hline CZ3 & $y=46.207-0.006 x$ & $0.5996^{\mathrm{ns}}$ & 7.974 \\
\hline \multicolumn{4}{|c|}{ 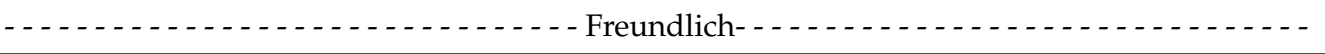 } \\
\hline Soil & $y=2.289+0.574 x$ & $0.9868 * *$ & $1.07 \times 10^{-3}$ \\
\hline $\mathrm{CZ}$ & $y=1.360+0.927 x$ & $0.9975^{* *}$ & $2.97 \times 10^{-4}$ \\
\hline CZ1 & $y=2.221+0.600 x$ & $0.9882 * *$ & $9.97 \times 10^{-4}$ \\
\hline $\mathrm{CZ2}$ & $y=2.216+0.600 x$ & $0.9914^{* *}$ & $7.18 \times 10^{-4}$ \\
\hline CZ3 & $y=2.176+0.615 x$ & $0.9894^{* *}$ & $9.09 \times 10^{-4}$ \\
\hline \multicolumn{4}{|c|}{ 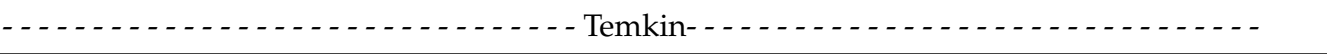 } \\
\hline Soil & $y=-3779.082+1527.953 x$ & 0.8630 * & 853 \\
\hline $\mathrm{CZ}$ & $y=-7605.247+2113.290 x$ & $0.9059 * *$ & 1266 \\
\hline CZ1 & $y=-4122.104+1583.930 x$ & $0.8666 *$ & 885 \\
\hline $\mathrm{CZ2}$ & $y=-4112.305+1572.963 x$ & 0.8777 * & 794 \\
\hline CZ3 & $y=-4304.231+1603.415 x$ & 0.8724 * & 871 \\
\hline
\end{tabular}

Note: Two ${ }^{* *}$ indicate significant relationship at $p=0.01$. One ${ }^{*}$ indicates significant relationship at $p=0.05 . \mathrm{ns}$ indicates not significant at $p=0.05$.

Table 17. Results from Langmuir and Freundlich isotherms for phosphorus adsorption by soil only, clinoptilolite zeolite only, and different amounts of clinoptilolite zeolite.

\begin{tabular}{|c|c|c|c|c|c|}
\hline \multirow[b]{2}{*}{ Treatment } & \multicolumn{3}{|c|}{ Langmuir } & \multicolumn{2}{|c|}{ Freundlich } \\
\hline & $\begin{array}{l}K_{L} \\
\left(\mu g g^{-1}\right)\end{array}$ & $\mathrm{q}_{\mathrm{m}}$ & $\begin{array}{l}\text { MBC } \\
\left(\mu g g^{-1}\right)\end{array}$ & $\begin{array}{l}K_{F} \\
\left(\mu g g^{-1}\right)\end{array}$ & $\frac{1}{n}$ \\
\hline Soil & 0.015 & 5000 & 75 & nd & nd \\
\hline CZ & 0.002 & 10,000 & 20 & nd & nd \\
\hline CZ1 & 0.013 & 5000 & 65 & nd & nd \\
\hline CZ2 & 0.013 & 5000 & 65 & nd & nd \\
\hline CZ3 & 0.012 & 5000 & 60 & nd & nd \\
\hline
\end{tabular}

Note: nd: not determined.

The soils with the CZ (CZ1, CZ2, and CZ3) best fitted with Langmuir type 2 isotherm because of the lower $\chi^{2}$ value (Tables 18 and 19). Potassium adsorption data for soil only and $\mathrm{CZ}$ alone best fitted best with Freundlich due to the significant $\mathrm{R}^{2}$ and lower $\chi^{2}$ values (Table 19). Langmuir bonding energy constant (KL), maximum adsorption capacity (qm), and maximum buffering capacity $(\mathrm{MBC})$ of $\mathrm{K}$ adsorption were determined from Langmuir type 2 equations for CZ1, CZ2, and CZ3 (Table 20). 
Table 18. Fitting Langmuir type 1, 2, and 3 isotherms to the potassium adsorption data based on simple regression and Chi-square analyses results.

\begin{tabular}{|c|c|c|c|}
\hline Treatment & Regression Equation & $\mathbf{R}^{2}$ & $x^{2}$ \\
\hline \multicolumn{4}{|c|}{ 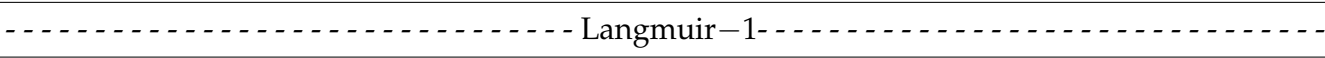 } \\
\hline Soil & $y=0.060+0.00006 x$ & $-0.1017^{\mathrm{ns}}$ & 0.018 \\
\hline $\mathrm{CZ}$ & $y=0.004-0.000001 x$ & $-0.3330 \mathrm{~ns}$ & $1.06 \times 10^{-3}$ \\
\hline CZ1 & $y=0.017+0.0001 x$ & $0.8537 *$ & $3.34 \times 10^{-3}$ \\
\hline $\mathrm{CZ2}$ & $y=0.011+0.0001 x$ & 0.8681 * & $2.28 \times 10^{-3}$ \\
\hline $\mathrm{CZ3}$ & $y=0.008+0.0001 x$ & 0.8479 * & $1.84 \times 10^{-3}$ \\
\hline \multicolumn{4}{|c|}{ 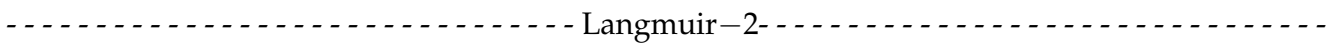 } \\
\hline Soil & $y=-0.00008+0.078 x$ & $0.9072^{* *}$ & $2.44 \times 10^{-4}$ \\
\hline $\mathrm{CZ}$ & $y=-0.00011+0.005 x$ & $0.8190 *$ & $2.68 \times 10^{-4}$ \\
\hline CZ1 & $y=0.00008+0.020 x$ & $0.9533^{* *}$ & $6.57 \times 10^{-5}$ \\
\hline $\mathrm{CZ2}$ & $y=0.00007+0.013 x$ & $0.9469 * *$ & $7.35 \times 10^{-5}$ \\
\hline CZ3 & $y=0.00005+0.010 x$ & $0.9349^{* *}$ & $8.74 \times 10^{-5}$ \\
\hline \multicolumn{4}{|c|}{ 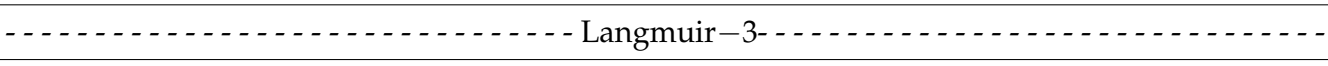 } \\
\hline Soil & $y=2765.355-4.025 x$ & $-0.3332^{\mathrm{ns}}$ & 5328 \\
\hline $\mathrm{CZ}$ & $y=2851.258+3.109 x$ & $-0.3183^{\mathrm{ns}}$ & 6845 \\
\hline $\mathrm{CZ1}$ & $y=6361.361-104.068 x$ & $0.2767^{\mathrm{ns}}$ & 928 \\
\hline $\mathrm{CZ2}$ & $y=6205.703-65.332 x$ & $0.3004^{\mathrm{ns}}$ & 4020 \\
\hline $\mathrm{CZ3}$ & $y=6019.559-44.594 x$ & $0.1712^{\mathrm{ns}}$ & 4119 \\
\hline
\end{tabular}

Note: Two ${ }^{* *}$ indicate significant relationship at $p=0.01$. One ${ }^{*}$ indicates significant relationship at $p=0.05 . \mathrm{ns}$ indicates not significant at $p=0.05$.

Table 19. Fitting Langmuir type 4, Freundlich, and Temkin isotherms to the potassium adsorption data based on simple regression and Chi-square analyses results.

\begin{tabular}{|c|c|c|c|}
\hline Treatment & Regression Equation & $\mathbf{R}^{2}$ & $x^{2}$ \\
\hline \multicolumn{4}{|c|}{ 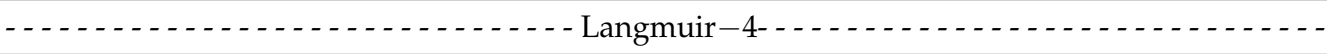 } \\
\hline Soil & $y=16.050-0.00002 x$ & $-0.3332 \mathrm{~ns}$ & 3.687 \\
\hline $\mathrm{CZ}$ & $y=261.133+0.004 x$ & $-0.3183^{\mathrm{ns}}$ & 105.637 \\
\hline CZ1 & $y=46.108-0.004 x$ & $0.2767^{\mathrm{ns}}$ & 9.595 \\
\hline CZ2 & $y=71.396-0.007 x$ & $0.3004^{\mathrm{ns}}$ & 17.375 \\
\hline CZ3 & $y=92.676-0.008 x$ & $0.1712^{\mathrm{ns}}$ & 28.994 \\
\hline \multicolumn{4}{|c|}{ 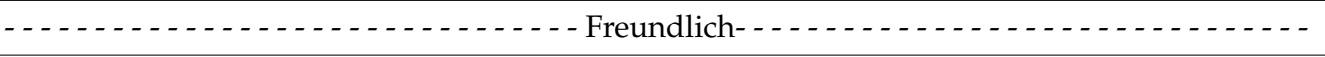 } \\
\hline Soil & $y=1.362+0.911 x$ & $0.7972 *$ & 0.025 \\
\hline $\mathrm{CZ}$ & $y=2.436+1.028 x$ & $0.8367 *$ & 0.020 \\
\hline CZ1 & $y=2.171+0.664 x$ & $0.8455 *$ & 0.015 \\
\hline $\mathrm{CZ2}$ & $y=2.349+0.643 x$ & $0.8360 *$ & 0.017 \\
\hline CZ3 & $y=2.423+0.657 x$ & 0.8291 * & 0.018 \\
\hline \multicolumn{4}{|c|}{ 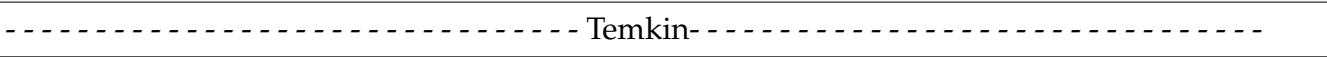 } \\
\hline Soil & $y=-6074.48+1752.61 x$ & $0.8527 *$ & 513.40 \\
\hline $\mathrm{CZ}$ & $y=-4445.60+3557.64 x$ & 0.9573 * & 293.35 \\
\hline CZ1 & $y=-4042.13+1668.18 x$ & 0.9085 * & 354.72 \\
\hline $\mathrm{CZ2}$ & $y=-3630.82+1799.48 x$ & $0.9101 *$ & 399.18 \\
\hline $\mathrm{CZ3}$ & $y=-3522.93+1953.97 x$ & 0.9130 * & 441.91 \\
\hline
\end{tabular}

Note: One ${ }^{*}$ indicates significant relationship at $p=0.05$. ns indicates not significant at $p=0.05$. 
Table 20. Results from Langmuir and Freundlich isotherms for potassium adsorption by soil only and different amounts of clinoptilolite zeolite.

\begin{tabular}{|c|c|c|c|c|c|}
\hline \multirow[b]{2}{*}{ Treatment } & \multicolumn{3}{|c|}{ Langmuir } & \multicolumn{2}{|c|}{ Freundlich } \\
\hline & $\begin{array}{c}K_{L} \\
\left(\mu g g^{-1}\right)\end{array}$ & $\mathrm{q}_{\mathrm{m}}$ & $\begin{array}{c}\text { MBC } \\
\left(\mu g g^{-1}\right)\end{array}$ & $\begin{array}{c}K_{F} \\
\left(\mu g g^{-1}\right)\end{array}$ & $\frac{1}{n}$ \\
\hline Soil & nd & nd & nd & 23.01 & 0.911 \\
\hline $\mathrm{CZ}$ & nd & nd & nd & 272.90 & 1.028 \\
\hline $\mathrm{CZ1}$ & 0.0040 & 12,500 & 50.00 & nd & nd \\
\hline $\mathrm{CZ2}$ & 0.0538 & 14,286 & 768.59 & nd & nd \\
\hline $\mathrm{CZ3}$ & 0.0500 & 20,000 & 1000.00 & nd & nd \\
\hline
\end{tabular}

Note: nd: not determined.

3.4. Nitrogen, Phosphorus, and Potassium Desorbed by Soil Only, Clinoptilolite Zeolite Only, and Soil with Different Amounts of Clinoptilolite Zeolite

Regardless of treatment, $\mathrm{N}$ and $\mathrm{P}$ desorption in relation to $\mathrm{N}$ and $\mathrm{P}$ concentrations were linearly related (Table 21). The fact the desorption amounts of $\mathrm{N}$ and $\mathrm{P}$ were higher for $\mathrm{CZ}$ only compared with soil only suggest that the $\mathrm{CZ}$ can release $\mathrm{N}$ and $\mathrm{P}$ into solution (Table 21).

Table 21. Linear relationships between the added amounts nitrogen, phosphorus, and potassium and their amounts desorbed.

\begin{tabular}{|c|c|c|}
\hline Treatment & Regression Equation & $\mathbf{R}^{2}$ Value \\
\hline \multicolumn{3}{|c|}{ 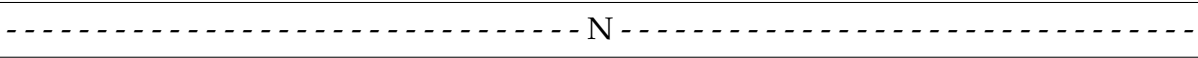 } \\
\hline Soil & $y=9.576+0.0034 x$ & $0.9758 * *$ \\
\hline $\mathrm{CZ}$ & $y=4.514+0.0276 x$ & $0.9995^{* *}$ \\
\hline CZ1 & $y=13.725+0.0070 x$ & $0.9781^{* *}$ \\
\hline $\mathrm{CZ2}$ & $y=16.388+0.0093 x$ & $0.9888^{* *}$ \\
\hline CZ3 & $y=17.402+0.0122 x$ & $0.9621 * *$ \\
\hline \multicolumn{3}{|c|}{ 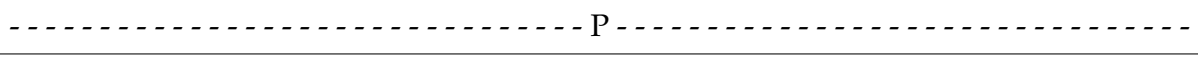 } \\
\hline Soil & $y=0.217+0.0037 x$ & $0.9678 * *$ \\
\hline $\mathrm{CZ}$ & $y=0.964+0.0043 x$ & $0.9855 * *$ \\
\hline CZ1 & $y=-0.400+0.0041 x$ & $0.9994^{* *}$ \\
\hline $\mathrm{CZ2}$ & $y=-0.069+0.0036 x$ & $0.9987^{* *}$ \\
\hline CZ3 & $y=-0.187+0.0038 x$ & $0.9964^{* *}$ \\
\hline \multicolumn{3}{|c|}{ 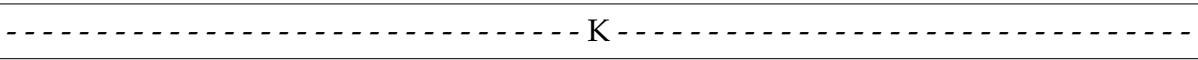 } \\
\hline Soil & $y=4.100+0.0045 x$ & $0.9559 * *$ \\
\hline $\mathrm{CZ}$ & $y=2.457+0.0014 x$ & $0.9333^{* *}$ \\
\hline CZ1 & $y=3.691+0.0043 x$ & $0.9719^{* *}$ \\
\hline $\mathrm{CZ2}$ & $y=2.633+0.0039 x$ & $0.9536 * *$ \\
\hline $\mathrm{CZ3}$ & $y=2.943+0.0031 x$ & $0.9506^{* *}$ \\
\hline
\end{tabular}

Note: ${ }^{* *}$ significant at $p=0.01$.

\section{5. pH Buffering Capacity of Clinoptilolite Zeolite}

Figure 2 summarizes the effects of soil only, $\mathrm{CZ}$ only, and soil with different amounts of $\mathrm{CZ}$ (CZ1, CZ2, and CZ3) on the $\mathrm{pH}$ buffering capacity of the soil. Regardless of treatment, the soil $\mathrm{pH}$ and added mmol $\mathrm{H}^{+}$related negatively (Figure 2 ). The $\mathrm{pH}$ and $\mathrm{pH}$ buffering capacity of the soil without clinoptilolite zeolite were lower because of the lower organic matter content and CEC of the soil (Table 22). 

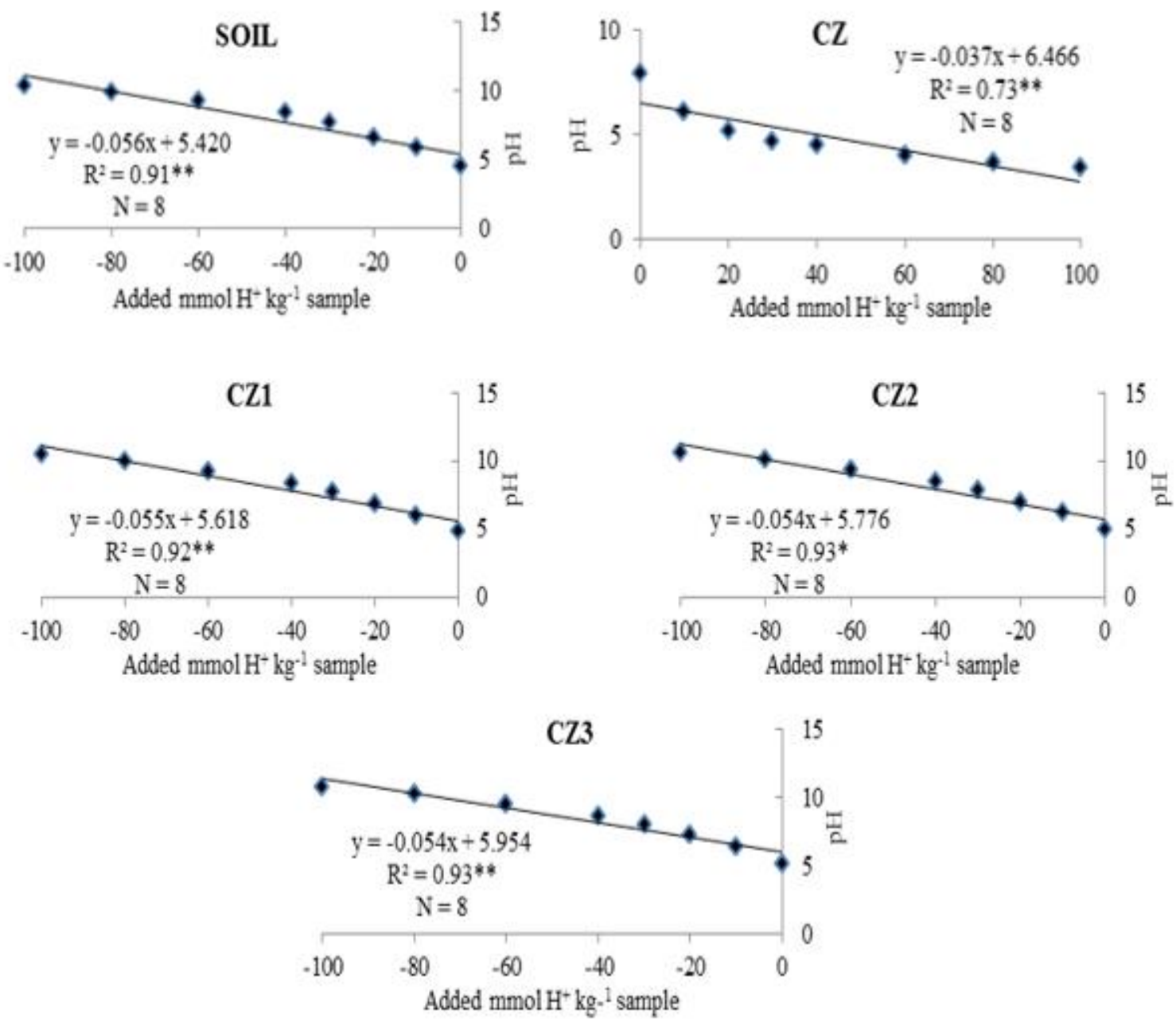

Figure 2. The linear between the added $\mathrm{mmol} \mathrm{H}^{+} \mathrm{kg}^{-1}$ sample and $\mathrm{pH}$ of suspension demonstrating $\mathrm{R}^{2}>0.73$ at $p=0.01$.

Table 22. Effects of soil only, clinoptilolite zeolite only, and soil with different amounts of clinoptilolite zeolite on the initial suspension $\mathrm{pH}$ and $\mathrm{pH}$ buffering capacity.

\begin{tabular}{|c|c|c|}
\hline Treatment & Initial pH & $\mathrm{pH}$ Buffering Capacity (mmol $\mathrm{H}^{+} \mathrm{kg}^{-1} \mathrm{pH}^{-1}$ Sample) \\
\hline Soil & $4.52( \pm 0.02)$ & 17.86 \\
\hline $\mathrm{CZ}$ & $7.87( \pm 0.03)$ & 27.03 \\
\hline CZ1 & $4.80( \pm 0.04)$ & 18.18 \\
\hline CZ2 & $4.96( \pm 0.04)$ & 18.52 \\
\hline CZ3 & $5.14( \pm 0.02)$ & 18.52 \\
\hline
\end{tabular}

\section{Discussion}

\subsection{Nitrogen, Phosphorus, and Potassium in Equilibrium Solution}

Increasing or no significant $\mathrm{N}$ or $\mathrm{P}$ or $\mathrm{K}$ contents in the equilibrium solution with increasing $\mathrm{CZ}$ rate demonstrate that the addition of $\mathrm{CZ}$ did not increase nutrient adsorption (Tables 4-6). The decreasing $\mathrm{N}$ or $\mathrm{P}$ or $\mathrm{K}$ contents in the equilibrium solution with increasing $\mathrm{CZ}$ rate indicates that the addition of the $\mathrm{CZ}$ increased these nutrients' adsorption (Tables 4-6). Compared with soil only, CZ only showed lower N, P, and K contents in the equilibrium solution of the no N, P, and $\mathrm{K}$ addition suggest that $\mathrm{CZ}$ had low exchangeable $\mathrm{NH}_{4}{ }^{+}, \mathrm{K}$, (Tables 4 and 6), and available P (Table 5). The decreasing rate of $\mathrm{N}$ or P or K remaining in the equilibrium solution with increasing amount of the $\mathrm{CZ}$ suggests that the use of increased the adsorption of these nutrients. However, the increasing or similar rate of $\mathrm{P}$ or $\mathrm{K}$ remaining in the equilibrium solution with increasing amount of the $\mathrm{CZ}$ suggests that the addition of the $\mathrm{CZ}$ in Typic Paleudults did not maximize adsorption of 
these nutrients (Table 7). In contrast to the use of natural zeolite as an ammonia adsorbent and $\mathrm{N}$ carrier, adsorption capacity of natural zeolite increases with the initial $\mathrm{NH}_{4}{ }^{+}$solution concentration [39]. Different type of zeolites affect adsorption capacity differently. For example, zeolite prepared from raw fly which has a slow adsorption capacity for $\mathrm{NH}_{4}{ }^{+}$, and its application is in fields with high concentration wastewater is limited [40]. In this present study, the different amounts of $\mathrm{CZ}$ used for $\mathrm{N}, \mathrm{P}$, and $\mathrm{K}$ adsorption affected the $\mathrm{N}, \mathrm{P}$, and $\mathrm{K}$ in equilibrium solutions and this observation is consistent with the findings of Tang et al. [40] who also reported that adsorption equilibrium between adsorbent and adsorbate is controlled by the adsorbent dosage.

\subsection{Nitrogen, Phosphorus, and Potassium Adsorption Isotherms}

The increased adsorbed $\mathrm{N}$ with the increasing rate of the $\mathrm{CZ}$ suggests that the $\mathrm{CZ}$ can improve $\mathrm{N}$ adsorption (Table 8). However, the similar adsorbed $\mathrm{P}$ or $\mathrm{K}$ with the increasing rates of the $\mathrm{CZ}$ indicates that the lowest rate of $\mathrm{CZ}$ was sufficient for the adsorption of $\mathrm{P}$ and $\mathrm{K}$ (Table 9). The higher cations $\left(\mathrm{NH}_{4}{ }^{+}\right.$and $\mathrm{K}^{+}$) and lower anion (phosphate) adsorption rates of the treatments with $\mathrm{CZ}$ was because of the negative charges of the $\mathrm{CZ}$ but the opposite was true for $\mathrm{P}$ which might have been repelled [41]. The increasing rates of $\mathrm{CZ}$ $(\mathrm{CZ1}<\mathrm{CZ2}<\mathrm{CZ} 3)$ increased cations $\left(\mathrm{NH}_{4}{ }^{+}\right.$and $\left.\mathrm{K}^{+}\right)$adsorption (Tables 8 and 10) because of the higher CEC of the $\mathrm{CZ}$. However, the $\mathrm{CZ}(\mathrm{CZ1}<\mathrm{CZ2}<\mathrm{CZ} 3)$ treatments reduced $\mathrm{P}$ adsorption because of the negative-negative charge coulumbic repulsive forces [42].

The higher adsorbed $\mathrm{N}$ or $\mathrm{P}$ or $\mathrm{K}$ in the $\mathrm{CZ}$ only treatment compared with soil only was due to the high CEC of the CZ whereas, the lower adsorbed P in CZ compared with soil only was due to anionic nature of $C Z$ (Tables 8-10). The coulumbic repulsive forces (same charge repulsion) of the $\mathrm{CZ}$ might have reduced the ability of the soil to adsorbed $\mathrm{P}$ [43]. Adsorbed $\mathrm{N}$ increased with increasing rates of $\mathrm{CZ}(\mathrm{CZ1}<\mathrm{CZ2}<\mathrm{CZ3})$ (Table 8). However, the increasing rates of $\mathrm{CZ}(\mathrm{CZ1}<\mathrm{CZ2}<\mathrm{CZ3})$ showed similar adsorbed $\mathrm{P}$ (Table 9). Adsorbed $\mathrm{K}$ was not affected by the increasing rates of $\mathrm{CZ}$ (CZ1 < CZ2 < CZ3) (Table 10). The higher rates of adsorption for $\mathrm{N}$ and $\mathrm{K}$ but not $\mathrm{P}$ were because of $\mathrm{CZ}$ has affinity for $\mathrm{NH}_{4}{ }^{+}$and $\mathrm{K}^{+}$(Table 11).

The fact that the experimental data of Langmuir $\mathrm{N}$ adsorption isotherms type 1, 2, and 3 exhibited negative intercepts (Table 12) suggest that the tested Langmuir isotherms type 1, 2, and 3 for $\mathrm{N}$ adsorption regardless of treatment contradict or are inconsistent with the underlying Langmuir assumption [37]. Freundlich isotherm suggests that the adsorbed $\mathrm{N}$ of the treatments occurred at heterogeneous adsorbent surface of samples (Table 13) [11]. High $\mathrm{K}_{\mathrm{F}}$ values of $\mathrm{CZ}$ alone, CZ1, CZ2, and CZ3 compared with soil alone suggests that the $\mathrm{CZ}$ has higher $\mathrm{N}$ adsorption capacity because of its higher CEC (Tables 13 and 14). In addition, the $1 / n>1$, regardless of treatment suggests that the $\mathrm{N}$ adsorption is not a favorable adsorption reaction [33]. Langmuir $P$ adsorption isotherm for soil only, $C Z$ only, CZ1, CZ2, and CZ3 (Tables 15-17) suggests that P was adsorbed by formation of a monolayer on the outermost surface of the adsorbent $[10,11]$. Irrespective of treatment, the highest bonding energy constant $\left(\mathrm{K}_{\mathrm{L}}\right)$ for $\mathrm{P}$ adsorption of soil only (Table 17) was due to precipitation of $\mathrm{P}$ by exchangeable $\mathrm{Al}^{3+}$ [41] because highly weathered tropical soils have anion exchange capacity to adsorb anions such as phosphates [42]. In addition, CZ alone, $\mathrm{CZ1}, \mathrm{CZ2}$, and $\mathrm{CZ3}$ showed lower $\mathrm{K}_{\mathrm{L}}$ compared with soil alone (Table 17) because of the high CEC of the $C Z$ and this means the negative charges of the afore-stated amendment might have repelled phosphates [41].

According to the literature, the maximum mass adsorbed at saturation conditions per mass unit of adsorbent in monolayer manners where, high $\mathrm{q}_{\mathrm{m}}$ requires less $\mathrm{P}$ saturation maximum mass adsorbed at saturation conditions per mass unit of adsorbent is referred to as maximum adsorption capacity $\left(\mathrm{q}_{\mathrm{m}}\right)$ [44]. Therefore, the higher maximum adsorption capacity $\left(\mathrm{qm}_{\mathrm{m}}\right)$ of $\mathrm{CZ}$ only relative to soil only (Table 17$)$ suggests that $\mathrm{CZ}$ only needs less $P$ for adsorbent saturation because the negatively charged exchange sites of the CZ only repelled P. Similarly, $\mathrm{qm}_{\mathrm{m}}$ of $\mathrm{CZ1}, \mathrm{CZ2}$, and CZ3 compared with soil alone shows that the $\mathrm{CZ}$ treatments $(\mathrm{CZ1}, \mathrm{CZ2}$, and $\mathrm{CZ3})$ required similar $\mathrm{P}$ to saturate the adsorbent. The 
lower $\mathrm{q}_{\mathrm{m}}$ of soil, $\mathrm{CZ1}, \mathrm{CZ2}$, and $\mathrm{CZ3}$ compared with $\mathrm{CZ}$ alone was because of the lower $\mathrm{P}$ content in the soil. Maximum buffering capacity (MBC) of $\mathrm{P}$ is the level at which adsorbent replenishes $P$ to sample solution because being inclined depletion [45] and this lend to support or add credence the reason why soil only significantly release $\mathrm{P}$ relative to $\mathrm{CZ}$ alone, CZ1, CZ2, and CZ3 (Table 17).

The treatment with the lower rate of $C Z(C Z 1)$ showed lower $K_{L}$ compared with those with higher rate (CZ2 and CZ3) because of the lower $\mathrm{K}$ content of the CZ but higher CEC (Tables 18-20). The affinity of the $C Z$ was higher at the higher rate of $C Z$. Increasing rate of $\mathrm{CZ}(\mathrm{CZ1}<\mathrm{CZ2}<\mathrm{CZ3})$ increased $\mathrm{q}_{\mathrm{m}}$ because lower amount of $\mathrm{K}$ was required to saturate the adsorbent. This was possible because of the inherent or native $\mathrm{K}$ of the $\mathrm{CZ}$ compared with soil alone. The increasing rate of $\mathrm{CZ}(\mathrm{CZ1}<\mathrm{CZ2}<\mathrm{CZ3})$ increased MBC because of the higher $C E C$ of the $C Z$. The higher $\mathrm{K}_{\mathrm{F}}$ value of $\mathrm{CZ}$ only compared with soil only was due to the high CEC of the CZ (Table 20 ). The $1 / n<1$ for soil only and $1 / n \approx 1$ for CZ only suggest favorable $\mathrm{K}$ adsorption (Table 20). The high $\mathrm{N}$ or P or K desorption rates of the CZ suggest they can temporary retain these nutrients although the CEC of the CZ is high. The lower K desorption rate (Table 21) but higher K adsorption capacity of CZ only (Table 20) compared with soil only suggests that sorption of $\mathrm{K}$ by $\mathrm{CZ}$ was more to absorption.

\subsection{Nitrogen, Phosphorus, and Potassium Desorption}

The increasing rate of the $\mathrm{CZ}(\mathrm{CZ1}<\mathrm{CZ} 2<\mathrm{CZ} 3)$ increased $\mathrm{N}$ desorption rate (Table 21) because of the lower affinity of the $\mathrm{CZ}$ for $\mathrm{NH}_{4}{ }^{+}$compared with $\mathrm{K}^{+}$[46]. The increasing rate of the $\mathrm{CZ}(\mathrm{CZ1}<\mathrm{CZ} 2<\mathrm{CZ} 3)$ reduced $\mathrm{P}$ and $\mathrm{K}$ desorption rate. The reduction in $\mathrm{P}$ desorption rate with the increasing $\mathrm{CZ}$ rate is consistent with the reduced maximum buffering capacity (Table 17) where, adsorbed $\mathrm{P}$ were not readily replenished during $\mathrm{P}$ depletion in the soil following the application of $\mathrm{CZ}$. Hence, $\mathrm{CZ}$ can be used to reduce $\mathrm{P}$ leaching. The increasing maximum buffering capacity (Table 20) with decreasing K desorption rate upon increasing $\mathrm{CZ}$ application suggests that $\mathrm{K}$ was more to absorption compared with adsorption, hence, the lower desorption. Absorption of $\mathrm{K}$ is further supported by the fact that the $\mathrm{CZ}$ demonstrated higher affinity for $\mathrm{K}^{+}$compared with other cations [47].

\subsection{Clinoptilolite Zeolite and Bekenu Series Soil Buffering Capacity}

The soil $\mathrm{pH}$ buffering capacity is typical of those of highly weathered soils (9 to $27 \mathrm{mmol} \mathrm{kg}^{-1} \mathrm{pH}^{-1}$ ) [34]. $\mathrm{pH}$ buffering capacity of the $\mathrm{CZ}$ only was higher than that of soil only due to the high $C E C$ of the $C Z$. The increasing rate of the $C Z(C Z 1<C Z 2<C Z 3)$ increased soil $\mathrm{pH}$ and $\mathrm{pH}$ buffering capacity because of the high $\mathrm{pH}$ and $\mathrm{pH}$ buffering capacity of the CZ (Figure 2 and Table 22). The $\mathrm{pH}$ buffering capacity soil only and the soil with different amounts of $\mathrm{CZ}$ were within the standard range of 10 to $100 \mathrm{mmol} \mathrm{H}^{+} \mathrm{kg}^{-1}$ $\mathrm{pH}^{-1}$ [33].

\section{Conclusions}

Clinoptilolite zeolite can increase $\mathrm{N}$ and $\mathrm{K}$ adsorption, $\mathrm{N}$ desorption rate, soil $\mathrm{pH}$, and $\mathrm{pH}$ buffering capacity irrespective of amount of $\mathrm{CZ}$ added. Additionally, it can reduce $\mathrm{P}$ adsorption and desorption of $\mathrm{P}$ and $\mathrm{K}$. Higher $\mathrm{K}$ adsorption with lower $\mathrm{K}$ desorption suggests that the $\mathrm{CZ}$ sorbs $\mathrm{K}$ effectively. The fact soil $\mathrm{N}$ and $\mathrm{K}$ adsorption and $\mathrm{P}$ desorption were improved, regardless of the amount of $\mathrm{CZ}$ added to the soil, indicates that the lowest rate of $\mathrm{CZ}$ was sufficient for the retention of total $\mathrm{N}$ and $\mathrm{K}$ and desorption of $\mathrm{P}$. Therefore, for the optimum rate, soil amended with $20 \mathrm{~g}$ of $\mathrm{CZ}$ is recommended to improve retention of soil total $\mathrm{N}$ and $\mathrm{K}$ and $\mathrm{P}$ desorption instead of $\mathrm{CZ}$ at 40 and $60 \mathrm{~g}$. Apart from the liming effect, CZ hindered re-acidification of amended Bekenu series soil by increasing soil $\mathrm{pH}$ buffering capacity. The limitation of the $\mathrm{N}, \mathrm{P}$, and $\mathrm{K}$ adsorption and desorption study is that it was not based on the conventional fertilization for $\mathrm{N}, \mathrm{P}$, and $\mathrm{K}$. However, the data have been tested in different isotherms such as Langmuir, Freundlich, and Temkin and these three isotherms confirmed the validity of $\mathrm{CZ}$ that could be used in $\mathrm{N}, \mathrm{P}$, and $\mathrm{K}$ retention and availability of $\mathrm{N}, \mathrm{P}$, and $\mathrm{K}$. 
Author Contributions: P.P. was responsible for conceptualization, investigation, writing, and original draft preparation, as well as for data analysis and visualization. O.H.A. was responsible for supervising, funding acquisition, project administration, experimental methodology, editing, and reviewing. L.O. was responsible for data arrangement and editing second draft. N.M.A.M. was also involve in funding acquisition and project administration. All authors have read and agreed to the published version of the manuscript.

Funding: This research was funded by Fundamental Research Grant Scheme (FRGS) grant code FRGS/2/2013/STWN03/UPM/02/6 from the Ministry of Higher Education, Malaysia.

Data Availability Statement: Not applicable.

Acknowledgments: The authors would like to acknowledge the Ministry of Higher Education, Malaysia for funding this research project through the Fundamental Research Grant Scheme (FRGS) grant code FRGS/2/2013/STWN03/UPM/02/6. Additionally, we acknowledge Universiti Putra Malaysia for the collaborative research.

Conflicts of Interest: The authors declare no conflict of interest.

\begin{abstract}
Abbreviations
N-nitrogen; P-phosphorus; K-potassium; CZ-clinoptilolite zeolite; CEC-cation exchange capacity; $\mathrm{NH}_{4}{ }^{+}$-ammonium; $\mathrm{NO}_{3}{ }^{-}$-nitrate; $\mathrm{C}$-carbon; EC-electrical conductivity; ANOVA-analysis of variance; CRD- completely randomized design; Fe-Iron, Al-aluminium; nd-not determine, NA-not applicable; $\mathrm{KCl}$-potassium chloride; $\mathrm{CaCl}$-calcium chloride.
\end{abstract}

\title{
References
}

1. Daković, A.; Tomašević-Čanović, M.; Rottinghaus, E.G.; Matijašević, S.; Sekulić, Z. Fumonisin B1 adsorption to octadecyldimetylbenzyl ammonium-modified clinoptilolite-rich zeolitic tuff. Microporous Mesoporous Mater. 2007, 105, 285-290. [CrossRef]

2. Latifah, O.; Ahmed, O.H.; Majid, N.M.A. Enhancing nitrogen availability from urea using clinoptilolite zeolite. Geoderma 2017, 306, 152-159. [CrossRef]

3. Ashman, M.R.; Puri, G. Essential Soil Science: A Clear and Concise Introduction to Soil Science; Blackwell Science Ltd.: England, UK, 2002.

4. Latifah, O.; Ahmed, O.H.; Majid, N.M.A. Enhancing nitrogen availability, ammonium adsorption-desorption, and soil pH buffering capacity using composted paddy husk. Eurasian Soil Sci. 2017, 50, 1-11. [CrossRef]

5. Jin, N.; Meng, C.X.; Hou, J. Preparation and characterization of merlinoite for potassium extraction from seawater. J. India Eng. Chem. 2014, 20, 1227-1230. [CrossRef]

6. Li, Z.; Anghel, I.; Bowman, R. Sorption of oxyanions by surfactant-modified zeolite. J. Dispers. Sci. Techol. 1998, 19, 843-857. [CrossRef]

7. Haggerty, G.M.; Bowman, R.S. Sorption of chromate and other inorganic anions by organo zeolite. Environ. Sci. Techol. 1994, 28, 452-458. [CrossRef] [PubMed]

8. $\mathrm{Li}, \mathrm{Z}$. Influence of solution $\mathrm{pH}$ and ionic strength on chromate uptake by surfactant-modified zeolite. J. Environ. Eng. 2004, 130, 205-208. [CrossRef]

9. Li, Z.; Beachner, R.; McManama, Z.; Hanlie, H. Sorption of arsenic by surfactant-modified zeolite and kaolinite. Microporous Mesoporous Mater. 2007, 105, 291-297. [CrossRef]

10. Okeola, F.O.; Odebunmi, E.O. Freundlich and Langmuir Isotherms parameters for adsorption of methylene blue by activated carbon derived from agro wastes. Adv. Nat. Appl. Sci. 2010, 4, 281-288.

11. Dada, A.; Olalekan, A.; Olatunya, A.; Dada, O. Langmuir, Freundlich, Temkin and Dubinin—Radushkevich isotherms studies of equilibrium sorption of $\mathrm{Zn}^{2+}$ unto phosphoric acid modified rice husk. J. Appl. Chem. 2012, 3, 38-45.

12. Dada, A.O.; Ojediran, J.O.; Olalekan, A.P. Sorption of $\mathrm{Pb}^{2+}$ from aqueous solution unto modified rice husk: Isotherms studies. Adv. Phys. Chem. 2013, 2013, 1-6. [CrossRef]

13. Foo, K.Y.; Hameed, B.H. Insights into the modeling of adsorption isotherm systems. Chem. Eng. J. 2010, 156, 2-10. [CrossRef]

14. Obiri-Nyarko, F.; Kwiatkowska-Malina, J.; Malina, G.; Kasela, T. Removal of lead and benzene from groundwater by zeolite and brown coal: Isotherm and kinetic studies. In Proceedings of the 4th International Conference on Environmental Pollution and Remediation, Prague, Czech Republic, 11-13 August 2014; pp. 1-7.

15. Ahmed, M.F.; Kennedy, I.R.; Choudhury, T.M.; Kecskes, M.L.; Deaker, R. Phosphorus adsorption in some Australian soils and influence of bacteria on the desorption of phosphorus. Commun. Soil Sci. Plant Anal. 2008, 39, 1269-1294. [CrossRef]

16. Bloom, A.J.; Frensch, J.; Taylor, A.R. Influence of inorganic nitrogen and pH on the elongation of maize seminal roots. Ann. Bot. 2006, 97, 867-873. [CrossRef] [PubMed]

17. Jusop, S.; Ishak, C.F. Weathered Tropical Soils the Ultisols and Oxisols; Universiti Putra Malaysia Press: Serdang, Malaysia, 2010. 
18. Ahmed, O.H.; Husni, A.; Ahmad, H.N.M.; Jalloh, M.B.; Rahim, A.A.; Majid, N.M.A. Enhancing the urea-N use efficiency in maize (Zea mays) cultivation on acid soils using urea amended with zeolite and TSP. Am. J. Appl. Sci. 2009, 6, 829-833. [CrossRef]

19. Peres-Caballero, R.; Gil, J.; Gondalez, J.L. The effect of adding zeolite to soils in order to improve the N-K nutrition of olive trees. Am. J. Aric. Biol. Sci. 2008, 2, 321-324.

20. Inglezakis, V.J.; Loizidou, M.D.; Grigoropoulou, H.P. Equilibrium and kinetic ion exchange studies of $\mathrm{Pb}^{2+}, \mathrm{Cr}^{3+}, \mathrm{Fe}^{3+}$, and $\mathrm{Cu}^{2+}$ on natural clinoptilolite. Water Res. 2002, 36, 2784-2792. [CrossRef]

21. Gruener, J.E.; Ming, D.W.; Henderson, K.E.; Galindo, C. Common ion effects in zeoponic substrates: Wheat plant growth experiment. Microporous Mesoporous Mater. 2003, 61, 223-230. [CrossRef]

22. McGilloway, R.L.; Weaver, R.W.; Ming, D.W.; Gruener, J.E. Nitrification in a Zeoponic substrate. Plant Soil 2003, 256, 371-378. [CrossRef] [PubMed]

23. Rehakova, M.S.; Cuvanova, M.; Dzivak, J.; Gaval'ovác, J. Agricultural and agrochemical uses of natural zeolite of the clinoptilolite type. Curr. Opin. Solid State Mater. Sci. 2004, 8, 397-404. [CrossRef]

24. Palanivell, P.; Ahmed, O.H.; Latifah, O.; Majid, N.M.A. Adsorption and desorption of nitrogen, phosphorus, potassium, and soil buffering capacity following application of chicken litter biochar to an acid soil. Appl. Sci. 2020, 10, 295. [CrossRef]

25. Tan, K.H. Soil Sampling, Preparation, and Analysis, 2nd ed.; CRC Press: Boca Raton, FL, USA, 2005.

26. Murphy, J.; Riley, R.I. A modified single solution method for the determination of phosphate in natural waters. Anal. Chim. Acta 1962, 27, 31-36. [CrossRef]

27. Keeney, D.R.; Nelson, D.W. Nitrogen-inorganic forms. In Method of Soil Analysis: Part 2; Page, A.G., Keeney, D.R., Baker, D.E., Miller, R.H., Rhoades, J.D., Eds.; Agronomy Monograph: Madison, WI, USA, 1962.

28. Paramananthan, S. Soil of Malaysia: Their Characteristics and Identification, Malaysia; Academy of Sciences Malaysia: Kuala Lumpur, Malaysia, 2000; Volume 1, pp. 11-125. ISBN 9839445065.

29. Bremner, J.M. Total Nitrogen. In Methods of Soil Analysis: Part 2; Black, C.A., Evans, D.D., Ensminger, L.E., White, J.L., Clark, F.F., Dinauer, R.C., Eds.; American Society of Agronomy: Madison, WI, USA, 1965; pp. 1149-1178.

30. Ming, D.W.; Dixon, J.B. Clinoptilolite in South Texas soils. Soil Sci. Soc. Am. J. 1986, 50, 1618-1622. [CrossRef]

31. Kithome, M.; Paul, J.W.; Lavkulich, L.M.; Bomke, A.A. Kinetics of ammonium adsorption and desorption by the natural zeolite clinoptilolite. Soil Sci. Soc. Am. J. 1998, 62, 622-629. [CrossRef]

32. Chowdhury, S.; Misra, R.; Kushwaha, P.; Das, P. Optimum sorption isotherm by linear and nonlinear methods for safranin onto alkali-treated rice husk. Bioremediat. J. 2011, 15, 77-89. [CrossRef]

33. Salarirad, M.M.; Behnamfard, A. Modeling of equilibrium data for free cyanide adsorption onto activated carbon by linear and non-linear regression methods. In Proceedings of the 2011 International Conference on Environment and Industrial Innovation (IPCBEE), Singapore, 26-28 February 2011; Volume 12, pp. 79-84.

34. Rowell, D.L. Soil Science: Methods and Applications; Longman Scientific \& Technical: Essex, UK, 1994.

35. Xu, R.; Zhao, A.; Yuan, J.; Jiang, J. pH buffering capacity of acid soils from tropical and subtropical regions of China as influenced by incorporation of crop straw biochars. J. Soils Sedim. 2012, 12, 494-502. [CrossRef]

36. Kissel, D.E.; Sonon, L.S.; Cabrera, M.L. Rapid measurement of soil pH buffering capacity. Soil Sci. Soc. Am. J. 2012, 76, 694-699. [CrossRef]

37. Castello, R.C.; Sullivan, D.M. Determining the $\mathrm{pH}$ buffering capacity of compost via titration with dilute sulphuric acid. Waste Biomass Valor 2014, 5, 505-513. [CrossRef]

38. SAS. SAS/STAT Software; SAS Institute: Cary, NY, USA, 2008.

39. Jelena, M.; Susanne, E.; Tore, K.; Vesna, R.; Nevenka, R. The use in grass production of clinoptilolite as an ammonia adsorbent and a nitrogen carrier. J. Serb. Chem. Soc. 2015, 80, 1203-1214.

40. Tang, H.; Xiaoyi Xu, X.; Wang, B.; Lv, C.; Shi, D. Removal of ammonium from swine wastewater using synthesized zeolite from fly ash. Sustainability 2020, 12, 3423. [CrossRef]

41. Onyango, M.S.; Masukume, M.; Ochieng, A.; Otieno, F. Functionalised natural zeolite and its potential for treating drinking water containing excess amount of nitrate. Water SA 2010, 36, 655-662. [CrossRef]

42. Kiurski, J.; Adamovic, S.; Oros, I.; Krstic, J.; Kovacevic, I. Adsorption feasibility in the Cr (total) ions removal from waste printing developer. Global NEST J. 2012, 14, 18-23.

43. Hinsinger, P. Bioavailability of soil inorganic P in the rhizosphere as affected by root-induced chemical changes: A review. Plant Soil 2001, 237, 173-195. [CrossRef]

44. Brady, N.C.; Weil, R.R. The Nature and Properties of Soils, 14th ed.; Pearson Education: Upper Saddle River, NJ, USA, 2008.

45. Ali, W.; Hussain, M.; Ali, M.; Mubushar, M.; Tabassam, A.R.M.; Mohsin, M.; Nasir, A. Evaluation of Freundlich and Langmuir isotherm for potassium adsorption phenomena. Int. J. Agric. Crop Sci. 2013, 6, 1048-1054.

46. Mehdi, S.M.; Sajjad, N.; Sarfraz, M.; Khalid, B.Y.; Hussan, G.; Sadiq, M. Response of wheat to different phosphatic fertilizer in varying textured salt affected soils. Pak. J. Appl. Sci. 2003, 3, 474-480. [CrossRef]

47. Fu, K.; Li, Z.; Xia, Q.; Zhong, T. Change and improving of ammonium exchange capacity onto zeolite in seawater. In Proceedings of the 2nd International Conference on Environmental Engineering and Applications, Shanghai, China, 19-21 August 2011; IACSIT Press: Singapore, 2011. 